\title{
AN INTERACTIVE TOOL TO INVESTIGATE THE INFERENCE PERFORMANCE OF NETWORK DYNAMICS FROM DATA
}

Veenadhar Katragadda

Thesis Prepared for the Degree of

\author{
MASTER OF SCIENCE
}

UNIVERSITY OF NORTH TEXAS

August 2012

APPROVED:

Yan Wan, Major Professor

Shengli Fu, Committee Member

Kamesh Namuduri, Committee Member

Murali Varanasi, Chair of the Department of Electrical Engineering

Costas Tsatsoulis, Dean of the College of Engineering

Mark Wardell, Dean of the Toulouse

Graduate School 
Veenadhar, Katragadda. An Interactive Tool to Investigate the Inference Performance of Network Dynamics from Data. Master of Science (Electrical Engineering), August 2012, 108 pp., 14 figures, references, 31 titles.

Network structure plays a significant role in determining the performance of network inference tasks. An interactive tool to study the dependence of network topology on estimation performance was developed. The tool allows end-users to easily create and modify network structures and observe the performance of pole estimation measured by Cramer-Rao bounds. The tool also automatically suggests the best measurement locations to maximize estimation performance, and thus finds its broad applications on the optimal design of data collection experiments. Finally, a series of theoretical results that explicitly connect subsets of network structures with inference performance are obtained. 
Copyright 2012

by

Veenadhar Katragadda 


\section{ACKNOWLEDGMENTS}

I would like to express my sincere gratitude to my advisor, Dr. Yan Wan for her guidance and support. As an advisor she always motivated me with her immense knowledge and made me enthusiastic to make my research successful. She is a great teacher and her classes are always fun and the way she present a topic is outstanding and her exams are always challenging and motivating. She is one of my favorite proffesors I met in my life.

Besides my advisor, I would like to thank Dr. Shengli Fu, Dr. Kamesh Namuduri, for their support as committee members of my thesis. A very special thanks to Dr. Xinrong Li for giving me insights of estimation thoery. I am so grateful for the support of my parents, my sister and my friends. Generally, I appreciate all the people I know for helping me grow up. 


\section{CONTENTS}

ACKNOWLEDGMENTS iii

CHAPTER 1. INTRODUCTION 1

1.1. Overview of Thesis Content 4

CHAPTER 2. BACKGROUND ON ESTIMATION THEORY AND CRAMER-RAO

BOUNDS 5

2.1. Estimation Theory 5

2.2. Cramer Rao Lower Bound 8

2.3. System Topology, Eigenvalues and Performance 10

CHAPTER 3. FUNCTIONAL DESCRIPTION OF INTERACTIVE TOOL 13

3.1. Options Panel 13

3.1.1. File Sub-Panel 13

3.1.2. Plot Options Sub-Panel 14

3.1.3. Settings Sub-Panel 15

3.1.4. Scaling Factor Sub-Panel 16

$\begin{array}{ll}\text { 3.2. } & \text { Plot Panel } \\ \end{array}$

$\begin{array}{ll}\text { 3.3. } & \text { Output Panel } \\ \end{array}$

3.3.1. System Dynamics Sub-Panel 17

3.3.2. Pole-Residue Representation Sub-Panel 18

3.3.3. Right Eigenvectors Sub-Panel 19

3.3.4. Fisher Information Sub-Panel 19 
3.3.5. Cramer-Rao Bounds for Poles Sub-Panel 19

3.3.6. Efficient Observation Placement Sub-Panel 20

$\begin{array}{lll}\text { CHAPTER 4. THEORETICAL BACKGROUND } & 21\end{array}$

$\begin{array}{ll}\text { 4.1. Fisher Information Matrix } & 21\end{array}$

4.2. Cramer-Rao Bounds for Pole Estimation 24

4.3. Effective Sensor Placement 24

4.3.1. Algorithm to Find the Effective Measurement Location 25

CHAPTER 5. SOME GRAPHICAL RESULTS ON ESTIMATION PERFORMANCE FOR SUBSETS OF NETWORK STRUCTURES 29

CHAPTER 6. CONCLUDING REMARK AND FUTURE WORK 36

APPENDIX A. MATLAB IMPLEMANTATION OF THE TOOL USING GUIDE-GUI MAIN FUNCTION 37

APPENDIX B. MATRIX FUNCTION: RETURNS DIFFERENT OUTPUTS IN GUI

APPENDIX C. OTHER FUNCTIONS REQUIRED FOR PLOTTING NETWORKS 100

$\begin{array}{ll}\text { C.1. PLOT GRAPH FUNCTION } & 101\end{array}$

C.2. GET NEAREST VERTEX FUNCTION 102

$\begin{array}{ll}\text { C.3. MAKE XY FUNCTION } & 103\end{array}$

$\begin{array}{ll}\text { C.4. GET POINT FUNCTION } & 103\end{array}$

$\begin{array}{lr}\text { BIBLIOGRAPHY } & 105\end{array}$ 


\section{CHAPTER 1}

\section{INTRODUCTION}

Systems have become structurally more complex and larger in size in a variety of applications, such as autonomous network control, transportation management, sensor networking, and power network surveillance, among others. Many of these systems are characterized by interconnected network topologies. While extensive advances have been achieved in the analysis, evaluation, and design of these networked systems, the inference of network dynamics/structure from data has just started to gain attention $[6,24,17]$. This growing interst is motivated by the fact that network inference tasks are critical for a range of design and control problems in networks. For instance, epidemic control relies on the inference of propagation dynamics from noisy outbreak data [28]. Genetic engineering is contingent upon the identification of gene regulatory networks from experimental microarray data $[18,17,4]$. Securing power networks is concerned with designing power network structure that is difficult for malicious attackers to estimate the configuration and dynamics of power systems from local observations (so as to disturb the network or to steal power) [12]. These network inference applications have motivated two critical research needs: 1) designing smart data-collection experiments to better understand a network's behavior, and 2) securing a networked system effectively against malicious probings and attacks. I note that from dynamical systems point of view, security is concerned with preventing attackers from using observation data to detect a system's internal dynamics [30].

I find that the key step to address the above two needs is to understand the pivotal factors impacting the performance of inference tasks in networks. This knowledge can then be utilized to design strategies to enhance security or surveillance capability. Previous ef- 
forts suggested that network topology has significant impact on the performance of inference tasks from noisy observation data [27]. In particular, estimation performance of system pole locations - as critical indicators of network dynamics - can be closely tied to network structure. However, mathematically capturing this structure dependence is nontrivial for general networks, as this problem is fundamentally concerned with directly relating eigenvectors, or equivalently zero-locations with network structure characteristics.

Computer-aided tools can significantly complement analytical limitations by providing automated analysis and design. The automated solutions can in turn provide rich insights to analytical discoveries. As such, in this thesis, I construct an interactive graphical user interface (GUI) tool that allows end users to easily modify a network's structure and observe the impact of such structural change on inference performance [15]. The tool is available to download at [1] with the interface shown in Figure 1.1. This stand-alone GUI tool has the following features:

1) End users can choose between reading the network structure from a file or directly drawing the network's graph structure in a plot area.

2) End users can select measurement noise levels and actuation and measurement locations to set up the network inference tasks. The tool then automatically displays the inference performance expressed in terms of Cramer-Rao bounds (CRB).

3) For any designated network structure, the tool displays the best measurement locations to observe network dynamics (e.g., with the tightest CRB). This information helps with the smart design of experiments to maximize inference performance.

4) End users can instantaneously observe the impact of structure change on inference performance, by directly modifying the graph structure through e.g., adding/removing nodes/edges, and dragging nodes. 


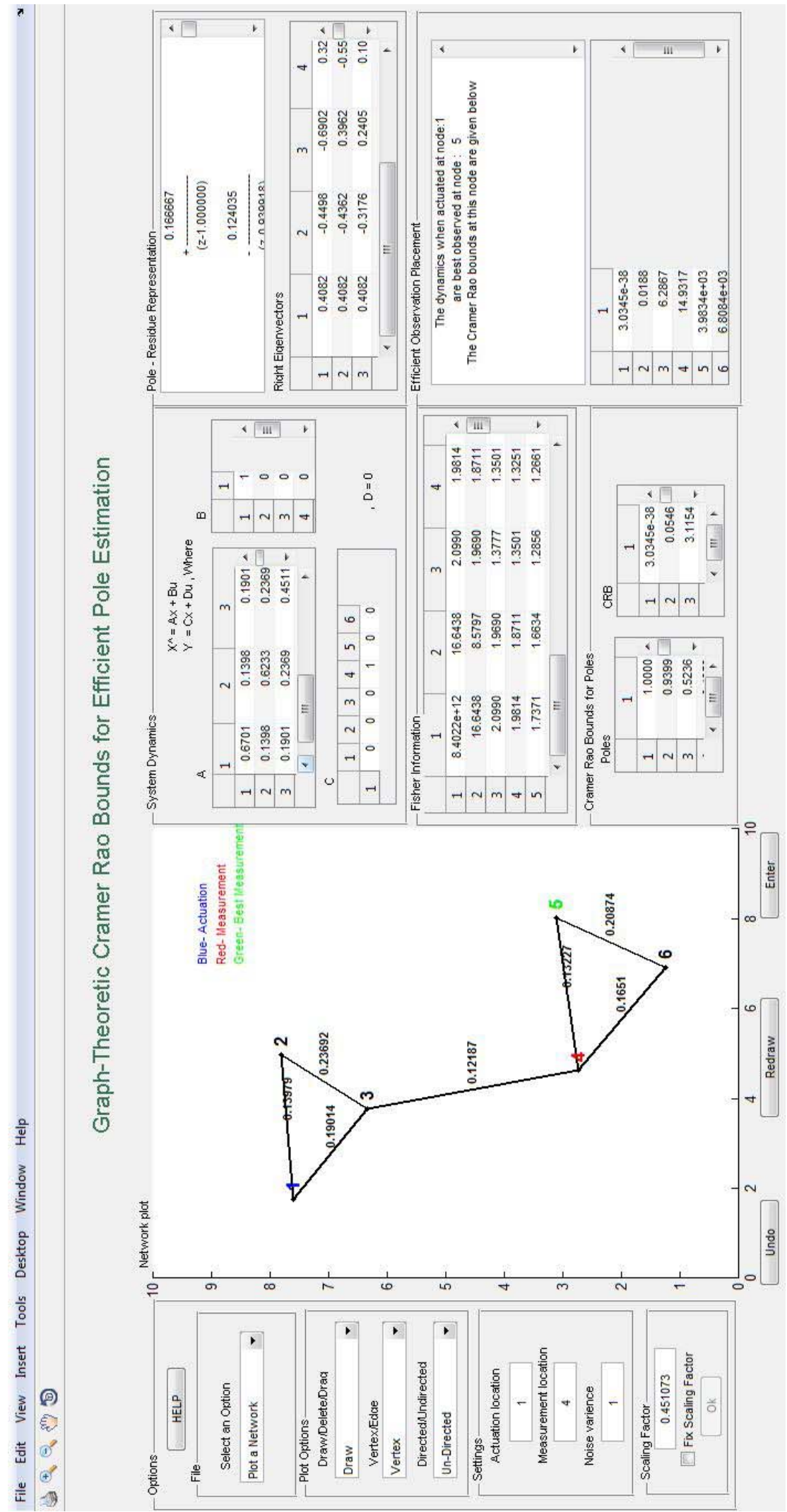

Figure 1.1. Interactive tool: Graphical User Interface 
In this thesis, I illustrate the functionalities of the tool, the theoretical background and algorithms for constructing the tool, and the analytical development of results obtained using the tool. In particular, I focus on the inference of system pole locations from noisy measurement data, and utilize the tool to help with: 1) evaluating the impact of network structural change on inference performance measured by $C R B, 2)$ identifying classes of network structures whose structural characteristics can be explicitly linked to inference performance, and 3) designing measurement locations to maximize inference performance. Building upon our previous development [27], I develop these results using meshed control theory, graph theory, and estimation theory. I envision that this interactive tool and resulting theoretical findings will provide us rich insights into structure-exploiting experimental design for smart data collection and strategies to enhance security.

\subsection{Overview of Thesis Content}

In this thesis, I illustrate the functionality, construction and the use of the GUI tool in studying the relationship between network structure and estimation performance in terms of CRB. The thesis is organized as follows. In Chapter 2, I introduce the broad background on estimation theory that is related to the development of this work. Chapter 3 presents the functional description of the GUI. Chapter 4 presents the theoretical background behind the implementation of the tool. Chapter 5 contains examples on utilizing this tool for scientific discovery. In particular, the tool allows us to identify subclasses of graphs, for which I provide theoretical proofs on the explicit relationship of them with the estimation performance. Finally, Chapter 6 includes a brief conclusion and future work. 
CHAPTER 2

\section{BACKGROUND ON ESTIMATION THEORY AND CRAMER-RAO BOUNDS}

This chapter provides the background on estimation theory, Cramer-Rao bounds and dynamical system theory that allow us to investigate the relationship between estimation performance and network structure.

\subsection{Estimation Theory}

In this section, I review the theory behind estimators, estimation process and how estimations are achieved in several applications. Estimation theory is an important subject in the fields of statistics and signal processing. Modern estimation theory is applied in a variety of signal processing applications. The goal of estimation theory is to extract some parameters from noisy observations and provide optimal estimates of these parameters. The process of estimation is different for different estimators. Estimator is a standard or a rule for measuring an estimate of a quantity based on observed data [5].

Estimators can be classified into two categories: point estimators and interval estimators. A point estimator yields a single value and the estimation involves calculating a single statistic which is the best estimate of the unknown parameter using the sample data. Minimum variance un-biased estimators (MVUE), best linear unbiased estimator (BLUE), minimum mean squared error estimator (MMSE) and maximum likelihood estimator (MLE) are the methods to derive point estimators [5].

Estimation error, Cramer-Rao bound and bias are typical metrics to evaluate the performance of point estimators [25]. The optimality of an estimate is determined by the estimation error which is the estimate itself minus the actual or true value of the parameter $e=\hat{\theta}-\theta$. If this error is high then the estimated value is not optimal and in contrast if the 
error is zero then the estimate is a perfect optimal one. The variance on estimation error of a unknown parameter is defined by a lower bound called Cramer-Rao bound (CRB). A detailed discussion on CRB is given in the section 2.2. Bias is defined as the difference between the expected value of the parameter that is to be estimated and it's true value. If $\hat{\theta}$ serves as an estimator to an unknown parameter $\theta$ then,

$$
\operatorname{Bias}[\hat{\theta}]=\mathbb{E}[\hat{\theta}]-\theta=\mathbb{E}[\hat{\theta}-\theta]
$$

If bias is zero then the estimator is un-biased. The estimator which is un-biased and having minimum. An estimate is said to be consistent if the mean-squared estimation error tends to zero as the number of observations increases $\lim _{L \rightarrow \infty} \mathbb{E}\left[e^{T} e\right]=0[16]$ and the estimator with this behavior is asymptotically unbiased. The estimator which has minimum varience and bias $=0$ (unbiased) is called minimum varience unbiased estimator (MVUE).

Interval estimator results in a range of possible values for an unknown parameter using the sample data. Single-valued result obtained from a point estimator can be expressed as a single function of a vector, whereas in interval estimators the results can be a range of multiple functions. I am not reviewing the details on interval estimator, as in this thesis, I am only interested in finding an MVU estimator, which falls into the category of point estimators.

The general estimation process is given by Figure 2.1. Major steps include:

- Determine probability distribution function (PDF) of the observed data. The PDF shows the dependence of the data on the parameters to be estimated.

- Develop an estimator for the above data model.

- Computer simulations can be done to test optimality and performance of the estimator. 


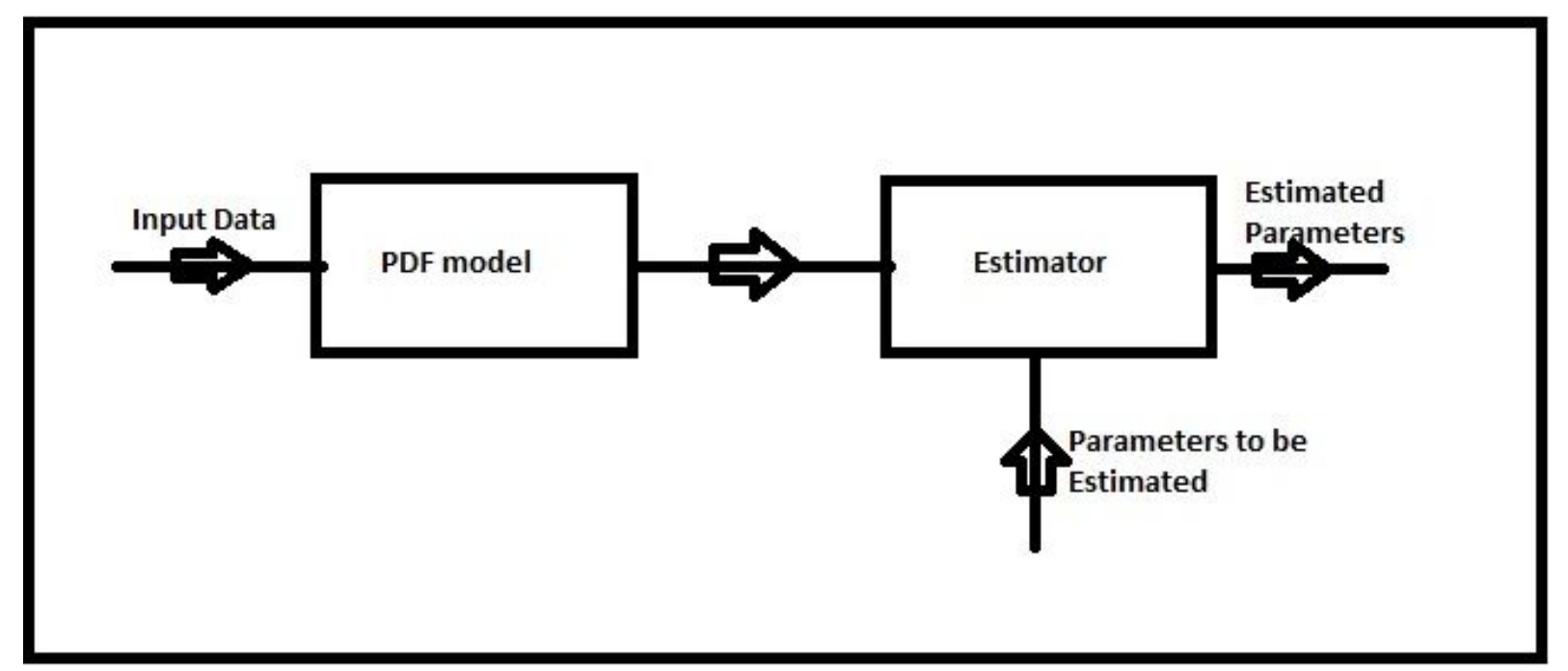

Figure 2.1. General estimation process

Estimation is performed in several applications including Radar, Sonar, Speech processing, Biomedicine, Control and Seismology. In all of these applications it is important to estimate a parameter or group of parameters based upon the experimental data which contains noisy random components. In Radar and Sonar, the interested parameters are the range and velocity of a target. In speech recognition, the parameter to be estimated can be some letter or word which is matched with the words already stored in the memory. In seismology the parameter to be estimated can be the distance of the underground oil deposit [16].

Pole estimation has its own significance in different applications. For example in signal processing, the poles of the harmonics are estimated to find the performance of detecting and tracking in a multi-channel setup [14]. The estimation in this work is performed using the total least square technique. The performance of the maximum likelihood estimator (MLE) estimating the time-of-arrival, amplitude parameters and poles from a noisy signal is studied in [9]. The work is motivated by underground acoustic testing where in the arrival time of the signal is unknown because of uncertainties. In this thesis, I am interested in developing 
a tool (refer Chapter(3) for details on the tool) for studying the relation between network structure and the performance of estimator, estimating poles of the network. I find CRB's for this study on estimation performance (refer 2.2 for background on CRB).

\subsection{Cramer Rao Lower Bound}

In the previous section, I discussed that the minimum variance unbiased estimator is always an efficient estimator. In practice, all the estimators are not MVU and sometimes there can be a possibility where no MVU estimator exsists. The non-existence of MVU estimator is shown in Figure 2.2. I can see that for $\theta<\theta_{0}$, the estimator $\theta_{2}$ has low variance and for $\theta>\theta_{0}$, the estimator $\theta_{3}$ has low variance. Therefore, it is undecidable for an optimal estimator or there is no MVU estimator. Cramer Rao Bound (CRB) is the tool which helps in finding a MVU estimator. If an estimator reaches this bound, it is MVU and hence an optimum estimator.

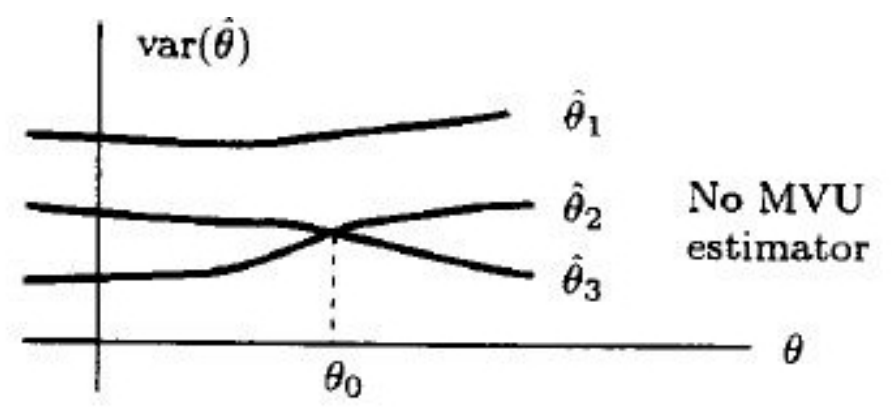

Figure 2.2. Variance of different estimators (From [16] topic: Existence of MVU estimator).

The CRB inequality or information inequality establishes the lower limit on how much information about an unknown probability distribution parameter, a set of measurements carries. In other words, it establishes the lower limit on variance of an estimator, estimating an unknown parameter $\theta$ having probability distribution $p(x ; \theta)$. CRB says that the lower limit on variance of unbiased estimator is at least the inverse of the Fisher information, $I(\theta)$ 
(equation (4)) [20]. Fisher information is the variance of the score $(V)$. Score is defined as the gradient (partial derivative) with respect to unknown parameter $\theta$ of the natural logarithm of likelihood function [11], given in equation (2).

$$
V=\frac{\partial}{\partial \theta} \log L(\theta ; M)=\frac{1}{L(\theta ; M)} \frac{\partial L(\theta ; M)}{\partial \theta}
$$

where $L$ is the Likelihood function, $M$ is the observation and $\theta$ is the unknown parameter.

To prove the inequality condition of CRB, let's assume $g$ as an unbiased estimator and $V$ is the score, then from Cauchy-Schwartz inequality [16] I have,

$$
(\mathbb{E}[(V-\mathbb{E}[V])(g-\mathbb{E}[g])])^{2} \leq \mathbb{E}\left[(V-\mathbb{E}[V])^{2}\right] \mathbb{E}\left[(g-\mathbb{E}[g])^{2}\right]
$$

As $g$ is an Unbiased estimator $\mathbb{E}[g]=\theta$ further simplifying equation(3) will result equation(4) (See [16] Topic: Cramer Rao bound, for entire proof).

$$
\operatorname{Var}(g) \geq \frac{1}{I(\theta)}
$$

where $I(\theta)=\mathbb{E}\left[V^{2}\right]=-\mathbb{E}\left[\frac{\partial^{2} \ln p(M ; \theta)}{\partial \theta^{2}}\right]$ is the Fisher Information.

The conditions under which the bound is valid is, [11]

- The bound is only valid to un-biased estimators while the biased estimators violate the lower bound.

- Maximum Likelihood Estimators (MLE) achieve the lower bound as the size of the observation data increases.

- In practice the bound may be unreachable. 


\subsection{System Topology, Eigenvalues and Performance}

Our efforts on developing an interactive tool is to study the connection between the graph topology and the estimation performance of the system. This section explains the relation between the system topology, eigenvalues and overall performance of the system. Several works have applied parameter estimation and system identification to study genetic networks in biological motivated applications, but the need for study on inference based on network/graph topology was commented [4]. Network structure plays an important role in studying inference performance. The topology of the network specifies about where in the network, measurements are to be made for definite inference of dynamics. Studying on the topological structure of the networks is encouraged in several fields. In Medicine, study on the brain regions which are interconnected by white matter tracts where in the network topology is important to understand the information sharing between brain regions and observe data for studying brain related diseases [13]. The work suggests role of network topology in studying the disease Schizophrenia. In computer security, analyzing the vulnerability issues based on graph structure are widely studied and several tools are developed which can detect network intrusion[21].

Now let us study the importance of poles in inference performance. Poles are the critical parameters indicating the performance and stability of the system. In this work, MLE of poles of single input single output (SISO) system from noisy data is considered (see chapter 4 for insights). The system dynamics are related to pole- residue or pole-zero form, which are shown in equations (5) and (6) [29].

$$
H[z]=\sum_{i=0}^{n-1} \frac{A_{i}}{z-p_{i}}
$$


where $A_{i}$ are the residues and $p_{i}$ are the poles.

$$
H[z]=\frac{\prod_{i=1}^{m} z-z_{i}}{\prod_{i=0}^{n-1} z-p_{i}}
$$

where $z_{i}$ are the zeros and $p_{i}$ are the residues. The location(value) of the pole determines the performance of the system. The following instances where poor performance of the system is observed are:-

- If the pole is nearer to zero, then numerator and the denominator of the transfer function given in equation(6) are nearly equal and so they get cancelled, resulting in poor dynamics and performance.

- If the residue $A_{i}$ is nearly zero, then from the equation(5) the numerator goes nearly zero resulting poor dynamics.

- If poles are repeated, then it is hard to study the behaviour of the system and hence the performance of the system cannot be analyzed.

In biological applications, modeling of virus spread is related to the network structure and the allocation of the resources in these networks are studied for minimizing the virus spread [28]. In this work, the network structure relating to the spread, determines the system matrices whose elements contains all the network interactions. The largest eigenvalue of the system matrix specifies the rate of expansion/contraction of the spread. In mathematical terms, minimizing the spread of the virus is to minimize the dominant eigenvalue. In our work, the system matrix which is related to the network structure is stochastic. The largest eigenvalue of the system matrix is always ' 1 ' because of it's stochastic nature. As already discussed, if the poles are repeated, one cannot infer much about the estimation performance. So, I will consider the study on inference of estimation performance at second largest eigenvalue. Similar work 
where in study on the second largest eigenvalue which accounts for the convergence rate of the network to infer consensus from network structure was done in [26]. Thus the system topology and the eigenvalues are critical in determining the estimation performance. 
CHAPTER 3

\section{FUNCTIONAL DESCRIPTION OF INTERACTIVE TOOL}

In this chapter, I will describe in detail the functionality and interface of the GUI tool. As this tool is designed to assist with studying a network structure's impact on estimation performance, I integrate the following features into the tool: 1) The graph capturing network structure is displayed to allow an intuitive and visualized observation; 2) End users can directly modify the network structure through dragging nodes or adding/deleting edges/nodes and observe the change of estimation performance automatically; and 3) The best measurement location is automatically generated for any designated actuation locations.

The interface of the tool is composed of three parts: 1) the Options panel where end users can set up the network for estimation performance analysis, 2) the Plot panel where the graph capturing network structure is displayed, and 3) the Output panel where the system dynamics and a variety of information to help with interpreting estimation performance is displayed. Here let us describe each of these three panels in detail.

\subsection{Options Panel}

The Options panel allows users to select various input modes and set up the network for estimation performance analysis. This panel includes four major sub-panels: the File subpanel, the Plot Options sub-panel, the Settings sub-panel and the Scaling Factor sub-panel.

\subsubsection{File Sub-Panel}

The File sub-panel allows end-users to select the source of network and save the current network structure. The drop-down menu has three options:

- Plot a Network: This is the default option. When this option is chosen, the user can draw a new network structure in the plot area. 
- Open Saved Network: This option allows the user to load an existing network structure into the GUI. After the network structure is loaded, the associated graph will appear in the plot area, and the user can then freely modify it. The network structure is stored in a *.mat format. In particular, the *.mat file contains two matrices: NodeLocation $\in R^{n \times 2}$ and Adjacency $\in R^{n \times n}$. Matrix NodeLocation stores the $x$ location (first column) and $y$ location (second column) of each node respectively. Matrix Adjacency is the adjacency matrix of the network, storing the connectivity information among the nodes. For instance, if there is a directed edge from vertex $i$ to $j$, then the Adjacency matrix has a value ' 1 ' at the $i$ th row and $j$ th column entry, denoted as Adjacency $i_{i j}$. Similarly, if there is an undirected edge between two vertices $i$ and $j$, both the entries Adjacency ${ }_{i j}$ and $A_{d j a c e n c y}$ are ' 1 '.

- Save Current Network: This option allows the user to store the current network structure in the plot area into a *.mat file. The format of the file is discussed above.

\subsubsection{Plot Options Sub-Panel}

This sub-panel allows end users to create and modify a network structure in the plot area. The sub-panel has three drop-down menus, namely Draw/Delete/Drag, Vertex/Edge, and Directed/Undirected. Here let us discuss the functionality for each combination of the selections defined by the three drop-down menus.

- Draw + Vertex: This combination allows the user to draw nodes in the plot area. Once the mouse is clicked, a node appears in the plot area and is labeled with a natural number starting from 1 . The current $x$ and $y$ locations of the mouse appear at the top of the plot area to facilitate the creation of a desired structure.

- Delete + Vertex: With this combination, once the mouse is clicked, the vertex that is closest to the click and all edges connected to the vertex will disappear. 
- Draw + Edge + Directed/Undirected: At this mode, when the user presses the mouse close to one vertex $i$, moves it to close to another vertex $j$, and then releases it, an edge between $i$ and $j$ is drawn in the undirected case, and from $i$ to $j$ is drawn in the directed case. The analysis developed in this thesis is only on the undirected network structures.

- Delete + Edge + Directed/Undirected: Similar to the above case, the edge that is the closest to the line defined by the press-release actions will be deleted.

- Drag Nodes: At this mode, when the user presses a node, moves it to another location and then releases it, the node is dragged to the new location and all edges connected to the node are moved simultaneously. The dragging capability is particularly useful to study the impact of network structural change on estimation performance.

\subsubsection{Settings Sub-Panel}

This panel allows the user to define actuation locations, measurement locations, and also the variance of Gaussian noise that the network is subject to.

- Actuation Location: The actuation location specifies the index of the node at which the network is actuated. In particular, I assume that a unit impulse input is applied at this location. Impulse-type inputs are widely used to capture short-time disturbances to systems (such as power failure in power grids, heat short to rats in biological experiments, etc.) $[22,23]$.

- Measurement Location: This is where the system response is measured. I assume that the network is measured at only one location. Moreover, an infinite length of sampled measurement sequence is available, so as to obtain the maximum estimation performance available at this measurement location.

- Noise Variance: I assume that the measurement is corrupted by a Gaussian noise 
with the variance $\sigma^{2}$ specified here. As I will show in Section 4, the variance of noise scales proportionally with CRB.

\subsubsection{Scaling Factor Sub-Panel}

In this thesis, I consider typical spread dynamics [28], which requires the state matrix A to be a stochastic matrix that satisfies two properties: 1) row sum being 1 , and 2) all the elements being in the range of 0 and 1 (i.e., $0<A_{i j}<1$ ). To ensure this requirement, a scaling process is required to convert distance-based edge weights into probability-like state matrix entries. This sub-panel displays the automatically selected scaling factor, and also allows the user to change it or fix its value so as to allow a fair comparison of estimation performance among networks subject to topological change. This sub-panel include three parts.

- Display/Edit Box: This component serves as a display box, showing the current scaling factor when fix scaling factor is unchecked. In this case, the scaling factor is automatically determined. It also serves as an edit box and allows the user to type the scaling factor when Fix Scaling Factor is checked.

- Fix Scaling Factor Check-Box: When this check box is checked, the scaling factor maintains the same despite all structural changes; otherwise it is determined automatically for each particular structure instance. This function is particularly useful when the user wishes to study the impact of structural change on estimation performance. Keeping the same scaling factor allows a fair comparison among different network structures. If the current scaling factor violates the properties of state matrices (e.g., with entries beyond the range of 0 to 1 ), the tool pops up an error message saying "Further change is not allowed because of the scaling constraint". When this occurs, the user can type a valid scaling factor directly in the Display/Edit box. 
- Ok button: This button enables the typed scaling factor. All results are recalculated based upon the new scaling factor. The Ok button is only valid when fix scaling factor is checked.

\subsection{Plot Panel}

This panel includes the plot area and three buttons: Enter, Undo, and Redraw.

- Enter: After completing the network construction, pressing the Enter button displays the estimation performance results in the Output panel. At the mean time, the actuation, measurement, and best measurement locations are marked in blue, red, and green respectively. Moreover, edge weights are also displayed in the plot area.

- Undo: This button cancels the last change (e.g., draw/change/delete) to the network.

- Redraw: Pressing this button will pop up a window asking the user to delete the entire network and start with drawing a new network. Pressing Yes will clear everything and this operation cannot be undone.

\subsection{Output Panel}

This panel displays the network dynamics, and all results that assist users in assessing estimation performance. Specifically, the panel includes the following components:

\subsubsection{System Dynamics Sub-Panel}

The system dynamics associated with the network structure displayed in the plot area is represented in a state-space form (refer to [27] for more details):

$$
\begin{aligned}
& \mathbf{x}[k+1]=A \mathbf{x}[k]+\mathbf{B} u[k] \\
& y[k]=\mathbf{C x}[k]+w[k],
\end{aligned}
$$


where $\mathbf{x}[k] \in R^{n}$ are the state variables representing the responses at each of the $n$ nodes in the network, $u[k]$ is a unit impulse signal, and $y[k]$ represents the measurement signal. As there is only one actuation location in the network, I indicate the actuation location in the vector $\mathbf{B} \in R^{n \times 1}$. Specifically, if the actuation is located at node $i, \mathbf{B}$ is a vector with its ith entry equal to 1 , and all the other entries equal to 0 . Similarly, $\mathbf{C} \in R^{1 \times n}$ is a row vector, with its $j$ th entry being 1 representing the measurement location, while all the other entries being 0 . Moreover, $w[k]$ is the additive Gaussian noise with mean 0 and variance $\sigma^{2}$.

State matrix $A \in R^{n \times n}$ is a stochastic matrix that captures the network structure shown in the plot area. In particular, $A$ is calculated from the network graph in the following. The tool first calculates the weight matrix $W \in R^{n \times n}$ where $W_{i j}$ is the inverse of the distance between node $i$ and node $j$. The Laplacian matrix $L$ is then generated from $W$ in such a way that each row sum of $L$ equals 0 . In another words, the diagonal entries of $L$ equal the negative sum of all off-diagonal entries in the same row. Matrix $L$ is further scaled with a scaling factor $\delta$ such that all entries of $L$ are between 0 and 1 . The default scaling factor $\delta$ is given in Equation (8).

$$
\delta=\frac{1}{1+\max (\operatorname{diag}(L))},
$$

where $\operatorname{diag}()$ means placing the diagonal entries of $L$ into a vector. Finally, $A$ matrix is formed as $A=I-L$, where $/$ is the identity matrix.

\subsubsection{Pole-Residue Representation Sub-Panel}

The pole-residue representation of the system transfer function $H[z]$ is the key toward the Cramer-Rao analysis, as it separates system poles as estimation parameters (see the detailed discussion in Section 4 and $[27,19]$ ). In particular, the representation is in the 
following format:

$$
H[z]=\sum_{i=0}^{n-1} \frac{A_{i}}{z-p_{i}}
$$

where $A_{i}$ are the residues and $p_{i}$ are the poles of the network. All eigenvalues of $A$ lies between -1 and 1 , with the largest eigenvalue at 1 .

\subsubsection{Right Eigenvectors Sub-Panel}

The sub-panel displays the right eigenvector associated with each eigenvalue. As seen from Section 5, estimation performance studies rely on algebraic graph theory results that relate eigenvectors with structural change $[7,3,8]$.

\subsubsection{Fisher Information Sub-Panel}

This sub-panel displays the Fisher Information matrix $I(\theta)$. Fisher Information matrix specifies the level of uncertainty in the observation sequence $y$ with respect to the unknown parameter vector $\theta$ (see $[16,19]$ for the details).

$$
I(\theta)=-\mathbb{E}\left(\frac{\partial \ln p(y ; \theta)^{T}}{\partial \theta} \frac{\partial \ln p(y ; \theta)}{\partial \theta}\right)
$$

where $y$ is the observation signal, $p(y ; \theta)$ is the probability function of $y$ expressed in terms of $\theta,{ }^{T}$ represents matrix transpose, and $E()$ is the expectation operator.

\subsubsection{Cramer-Rao Bounds for Poles Sub-Panel}

Cramer-Rao bound specifies a lower bound on the variance of estimated parameter $\theta$. System poles and their associated CRB are displayed in this sub-panel. CRB is obtained by inverting the Fisher Information matrix as shown in Equation (11) [16]. 


$$
\operatorname{var}(\theta) \geq I(\theta)^{-1}
$$

\subsubsection{Efficient Observation Placement Sub-Panel}

Given a fixed actuation location, this sub-panel provides the best measurement location to observe the second largest eigenvalue, or named the Fiedler eigenvalue [7]. The Fiedler eigenvalue is a critical indicator of system dynamics and has received extensive study (see e.g., $[7,8,28,2,26])$. For instance, it captures the mixing speed of a markov chain [2], the convergence speed of consensus building [26], and the spread speed of an epidemic [28]. The CRB associated with this optimal observation is also displayed. 


\section{CHAPTER 4}

\section{THEORETICAL BACKGROUND}

In this chapter, I describe the theoretical foundations of the tool. In particular, I first show how the CRBs for pole estimations are obtained from the observation sequence. This analysis is based upon the pole-residue representation which relates system response (captured by the observation sequence) with pole locations. Moreover, in many applications, finding observation locations associated with the best estimation performance are interested. These locations represent the most effective places to ascertain system dynamics, or equivalently, the most vulnerable sites to release system dynamics if seized by adversaries. I thus also discuss the calculation of optimal measurement locations.

\subsection{Fisher Information Matrix}

In this section, I consider the system shown in Equation (7), and discuss how the Fisher information matrix for pole estimation can be obtained. Most of the analysis discussed here can be found in [27]. I summarize the results here for the completeness of our presentation.

I start from the pole-residue representation of system dynamics as shown in Equation (9). I denote the parameter of interest $\theta$ as a cascade of the parameters $A=\left[\begin{array}{llll}A_{0} & A_{1} & A_{2} & \ldots\end{array}\right]$ and $p=\left[\begin{array}{llll}p_{0} & p_{1} & p_{2} & \ldots\end{array}\right]$ in the pole-residue form. In particular, $\theta=\left[\begin{array}{ll}A & P\end{array}\right]$. Moreover, as the output sequence $y[k]$ can be written as [27]

$$
y[k]=\sum_{i=0}^{n-1} A_{i} p_{i}^{k}+w[k]
$$

we can easily obtain the Fisher information matrix as $[27,19]$ 
(13) $\quad F(\theta)=\frac{1}{\sigma^{2}} \frac{\partial y_{j}^{\top}}{\partial \theta} \frac{\partial y_{j}}{\partial \theta}=\frac{1}{\sigma^{2}}\left[\begin{array}{l}\frac{\partial y_{j}^{T}}{\partial A} \\ \frac{\partial y_{j}^{\top}}{\partial p}\end{array}\right]\left[\begin{array}{ll}\frac{\partial y_{j}}{\partial A} & \frac{\partial y_{j}}{\partial p}\end{array}\right]$

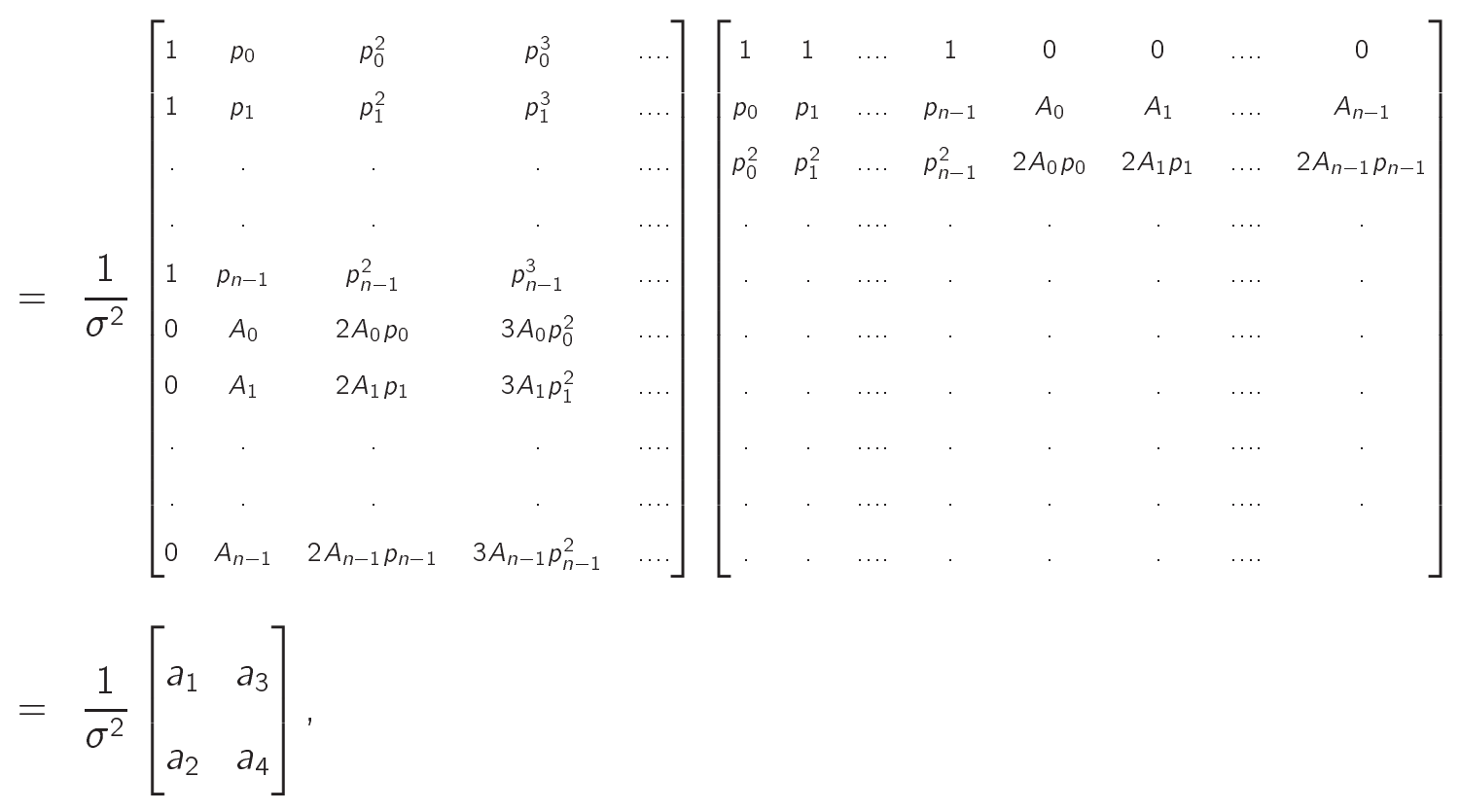

where

$$
\begin{aligned}
a_{1}= & {\left[\begin{array}{cccc}
\left(1+p_{0}^{2}+p_{0}^{4}+\ldots\right) & \left(1+p_{0} p_{1}+p_{0}^{2} p_{1}^{2}+\ldots\right) & \ldots & \left(1+p_{0} p_{n-1}+p_{0}^{2} p_{n-1}^{2}+\ldots\right) \\
\left(1+p_{0} p_{1}+p_{0}^{2} p_{1}^{2}+\ldots\right) & \left(1+p_{1}^{2}+p_{1}^{4}+\ldots\right) & \ldots & \left(1+p_{1} p_{n-1}+p_{1}^{2} p_{n-1}^{2}+\ldots\right) \\
\cdot & \ldots & \ldots \\
\left(1+p_{0} p_{n-1}+p_{0}^{2} p_{n-1}^{2}+\ldots\right) & \left(1+p_{1} p_{n-1}+p_{1}^{2} p_{n-1}^{2}+\ldots\right) & \ldots & \left(1+p_{n-1}^{2}+p_{n-1}^{4}+\ldots\right)
\end{array}\right] } \\
= & {\left[\begin{array}{cccc}
\frac{1}{1-p_{0}^{2}} & \frac{1}{1-p_{0} p_{1}} & \ldots & \frac{1}{1-p_{0} p_{n-1}} \\
\frac{1}{1-p_{0} p_{1}} & \frac{1}{1-p_{1}^{2}} & \ldots & \frac{1}{1-p_{1} p_{n-1}} \\
\cdot & \cdot & \ldots & \cdot \\
\frac{1}{1} & \frac{1}{1-p_{n-1} p_{1}} & \ldots & \frac{1}{1-p_{n-1}^{2}}
\end{array}\right] }
\end{aligned}
$$


$a_{2}=\left[\begin{array}{cccc}\left(p_{0} A_{0}+p_{0}^{2}\left(2 A_{0} p_{0}\right)+\ldots\right) & \left(p_{1} A_{0}+p_{1}^{2}\left(2 A_{0} p_{0}\right)+\ldots\right) & \ldots & \left(p_{n-1} A_{0}+p_{n-1}^{2}\left(2 A_{0} p_{0}\right)+\ldots\right) \\ \left(p_{0} A_{1}+p_{0}^{2}\left(2 A_{1} p_{1}\right)+\ldots\right) & \left(p_{1} A_{1}+p_{1}^{2}\left(2 A_{1} p_{1}\right)+\ldots\right) & \ldots & \left(p_{n-1} A_{1}+p_{n-1}^{2}\left(2 A_{1} p_{1}\right)+\ldots\right) \\ \cdot & . & \ldots & \\ \cdot & . & \ldots & \\ \left(p_{0} A_{n-1}+p_{0}^{2}\left(2 A_{n-1} p_{n-1}\right)+\ldots\right) & \left(p_{1} A_{n-1}+p_{1}^{2}\left(2 A_{n-1} p_{n-1}\right)+\ldots\right) & \ldots & \left(p_{n-1} A_{n-1}+p_{n-1}^{2}\left(2 A_{n-1} p_{n-1}\right)+\ldots\right)\end{array}\right]$

$(15)=\left[\begin{array}{cccc}\frac{A_{0} p_{0}}{\left(1-p_{0}^{2}\right)^{2}} & \frac{A_{0} p_{1}}{\left(1-p_{0} p_{1}\right)^{2}} & \cdots & \frac{A_{0} p_{n-1}}{\left(1-p_{0} p_{n-1}\right)^{2}} \\ \frac{A_{1} p_{0}}{\left(1-p_{1} p_{0}\right)^{2}} & \frac{A_{1} p_{1}}{\left(1-p_{1}^{2}\right)^{2}} & \cdots & \frac{A_{1} p_{n-1}}{\left(1-p_{1} p_{n-1}\right)^{2}} \\ \cdot & \cdot & \cdots & \cdot \\ \cdot & \cdot & \cdots & \cdot \\ \frac{A_{n-1} p_{0}}{\left(1-p_{n-1} p_{0}\right)^{2}} & \frac{A_{n-1} p_{1}}{\left(1-p_{n-1} p_{1}\right)^{2}} & \cdots & \frac{A_{n-1} p_{n-1}}{\left(1-p_{n-1}^{2}\right)^{2}}\end{array}\right]$

$a_{3}=\left[\begin{array}{ccc}\left(p_{0} A_{0}+p_{0}^{2}\left(2 A_{0} p_{0}\right)+p_{0}^{3}\left(3 A_{0} p_{0}^{2}\right)+\ldots\right) & \ldots & \left(p_{0} A_{n-1}+p_{0}^{2}\left(2 A_{n-1} p_{n-1}\right)+p_{0}^{3}\left(3 A_{n-1} p_{n-1}^{2}\right)+\ldots\right) \\ \left(p_{1} A_{0}+p_{1}^{2}\left(2 A_{0} p_{0}\right)+p_{1}^{3}\left(3 A_{0} p_{0}^{2}\right)+\ldots\right) & \ldots & \left(p_{1} A_{n-1}+p_{1}^{2}\left(2 A_{n-1} p_{n-1}\right)+p_{1}^{3}\left(3 A_{n-1} p_{n-1}^{2}\right)+\ldots\right) \\ & \ldots & \\ \cdot & \ldots & \\ \left(p_{n-1} A_{0}+p_{n-1}^{2}\left(2 A_{0} p_{0}\right)+p_{n-1}^{3}\left(3 A_{0} p_{0}^{2}\right)+\ldots\right) & \ldots & \left(p_{n-1} A_{n-1}+p_{n-1}^{2}\left(2 A_{n-1} p_{n-1}\right)+p_{n-1}^{3}\left(3 A_{n-1} p_{n-1}^{2}\right)+\ldots\right)\end{array}\right]$

$(16)=\left[\begin{array}{cccc}\frac{A_{0} p_{0}}{\left(1-p_{0}^{2}\right)^{2}} & \frac{A_{1} p_{0}}{\left(1-p_{0} p_{1}\right)^{2}} & \cdots & \frac{A_{n-1} p_{0}}{\left(1-p_{0} p_{n-1}\right)^{2}} \\ \frac{A_{0} p_{1}}{\left(1-p_{1} p_{0}\right)^{2}} & \frac{A_{1} p_{1}}{\left(1-p_{1}^{2}\right)^{2}} & \cdots & \frac{A_{n-1} p_{1}}{\left(1-p_{1} p_{n-1}\right)^{2}} \\ \cdot & \cdot & \cdots & \cdot \\ \cdot & \cdot & \cdots & \cdot \\ \frac{A_{0} p_{n-1}}{\left(1-p_{n-1} p_{0}\right)^{2}} & \frac{A_{1} p_{n-1}}{\left(1-p_{n-1} p_{1}\right)^{2}} & \cdots & \frac{A_{n-1} p_{n-1}}{\left(1-p_{n-1}^{2}\right)^{2}}\end{array}\right]$

and

$a_{4}=\left[\begin{array}{ccc}\left(A_{0}^{2}+\left(2 A_{0} p_{0}\right)^{2}+\left(3 A_{0} p_{0}^{2}\right)^{2}+\ldots\right) & \ldots & \left(A_{0} A_{n-1}+\left(2 A_{0} p_{0}\right)\left(2 A_{n-1} p_{n-1}\right)+\ldots\right) \\ \left(A_{0} A_{1}+\left(2 A_{1} p_{1}\right)\left(2 A_{0} p_{0}\right)+\left(3 A_{1} p_{1}^{2}\right)\left(3 A_{0} p_{0}^{2}\right)+\ldots .\right) & \ldots & \left(A_{1} A_{n-1}+\left(2 A_{1} p_{1}\right)\left(2 A_{n-1} p_{n-1}\right)+\ldots\right) \\ \cdot & \ldots & \\ \cdot & \ldots & \\ \left(A_{0} A_{n-1}+\left(2 A_{n-1} p_{n-1}\right)\left(2 A_{0} p_{0}\right)+\left(3 A_{n-1} p_{n-1}^{2}\right)\left(3 A_{0} p_{0}^{2}\right)+\ldots .\right) & \ldots & \left(A_{n-1}^{2}+\left(2 A_{n-1} p_{n-1}\right)^{2}+\left(3 A_{n-1} p_{n-1}^{2}\right)^{2}+\ldots .\right)\end{array}\right]$ 


$$
(17)=\left[\begin{array}{cccc}
\frac{A_{0}^{2}\left(1+p_{0}^{2}\right)}{\left(1-p_{0}^{2}\right)^{3}} & \frac{A_{0} A_{1}\left(1+p_{0} p_{1}\right)}{\left(1-p_{0}\right)^{3}} & \ldots & \frac{A_{0} A_{n-1}\left(1+p_{0} p_{n-1}\right)}{\left(1-p_{0} p_{n-1}\right)^{3}} \\
\frac{A_{0} A_{1}\left(1+p_{0} p_{1}\right)}{\left(1-p_{0} p_{1}\right)^{3}} & \frac{A_{1}^{2}\left(1+\left(p_{1}\right)^{2}\right)}{\left(1-p_{1}^{2}\right)^{3}} & \ldots & \frac{A_{1} A_{n-1}\left(1+p_{1} p_{n-1}\right)}{\left(1-p_{1} p_{n-1}\right)^{3}} \\
\cdot & & \ldots & \\
\cdot & & \ldots & \\
\frac{A_{0} A_{n-1}\left(1+p_{0} p_{n-1}\right)}{\left(1-p_{0} p_{n-1}\right)^{3}} & \frac{A_{1} A_{n-1}\left(1+p_{1} p_{n-1}\right)}{\left(1-p_{1} p_{n-1}\right)^{3}} & \ldots & \frac{A_{n-1}^{2}\left(1+p_{n-1}^{2}\right)}{\left(1-p_{n-1}^{2}\right)^{3}}
\end{array}\right]
$$

4.2. Cramer-Rao Bounds for Pole Estimation

The CRB for the estimate of parameter $\theta_{i}$ is the ith diagonal entry of the inverse of the Fisher information matrix:

$$
C_{i}=\left(F(\theta)^{-1}\right)_{i i}
$$

Of our particular interest, the CRB for the ith pole $p_{i}$ (denoted as $C\left(p_{i}\right)$ ) equals $C_{n+i+1}$. Because the calculation of CRB is involved with inverting a large matrix that is prone to be ill-conditioned, I instead find a lower-bound of the CRB. In particular, the CRB associated with the ith pole is bounded by [27]

$$
C\left(p_{i}\right) \geq\left(\frac{1}{\sigma^{2}} \frac{A_{i}^{2}\left(1+p_{i}^{2}\right)}{\left(1-p_{i}^{2}\right)^{3}}\right)^{-1}=\sigma^{2} \frac{\left(1-p_{i}^{2}\right)^{3}}{A_{i}^{2}\left(1+p_{i}^{2}\right)}
$$

The above result shows that CRB scales with the variance of Gaussian noise. Moreover, the residues play an important role in the performance of pole estimation. Generally, the poles associated with large residues are easier to estimate than the ones with smaller residues [27].

\subsection{Effective Sensor Placement}

In many sensor network applications such as bridge health monitoring and environmental monitoring, sensors are placed ad hoc. However, as noted recently, smartly choosing 


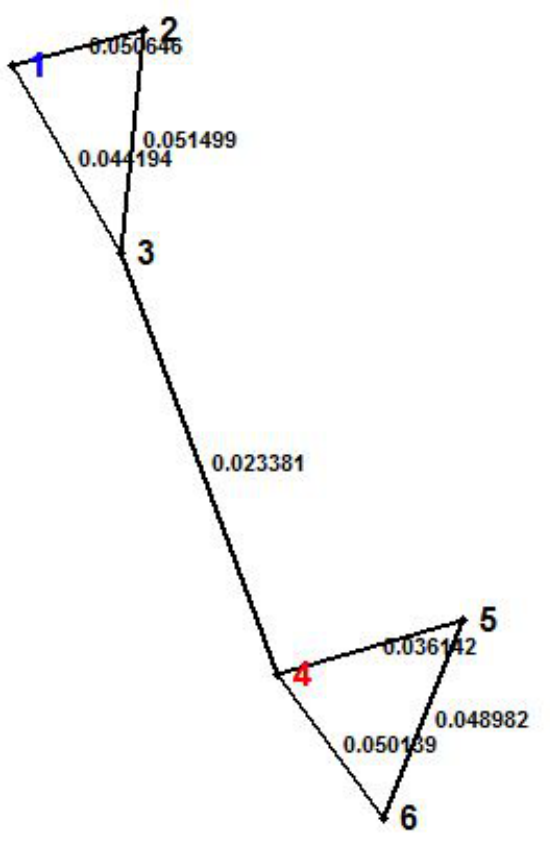

Figure 4.1. Example of input network structure.

sensor locations can significantly enhance estimation performance $[10,31]$. Our philosophy is that some basic understanding of network structure can lead to a structure-exploiting design of effective sensor locations.

In this tool, the best observation location is displayed for a fixed network structure and actuation location. The best observation location is achieved by looping through all possible locations and comparing the CRBs of the Fiedler eigenvalue associated with each location.

\subsubsection{Algorithm to Find the Effective Measurement Location}

As I relate the structure of the network to the discrete SISO system dynamics, The first step is to get the dynamics from the input structure and relate to the equation (7). 
$\% \%$ To get matrix $A \% \% \%$

Get the distance between all the $n$ vertices from the input graph

Find the edge weights by the inverting the above distances

Find the Laplacian Matrix $L$ whose elements are the edge weights

IF the elements of Laplacian matrix are $>1$ or $<0$

Scale the edge weights to be in the range $(0,1)$

\section{ENDIF}

Negate all the elements of $L$

Find the State matrix $A$ using $A=I-L$

$B$ and $C$ represents the actuation and measurement locations.

Get the $\mathrm{n}^{*} 1$ matrix $B$ and put ' 1 ' in the matrix at the actuation location. Rest of the elements are zero

Get the $1^{*}$ n matrix $C$ and put ' 1 ' in the matrix at measurement location. Rest of the elements are zero

$D=0$

$\% \%$ To get the Cramer - Rao bounds $\% \% \%$

Get the transfer function from the state-space form

Find the Poles and Residues from transfer function

The Fisher matrix is obtained using equation(13)

Find CRB by inverting the Fisher matrix (equation(18))

$\% \% \%$ To get the Efficient Cramer - Rao bounds \%\%\% 


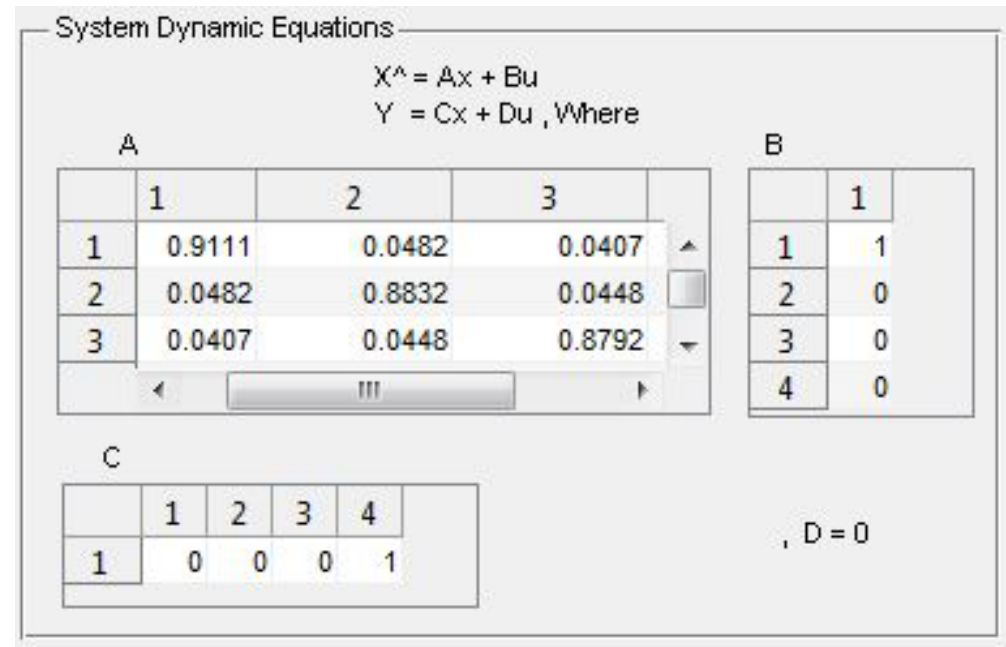

Figure 4.2. System dynamics.

\begin{tabular}{|c|c|c|c|c|c|c|c|c|}
\hline \multicolumn{9}{|c|}{ Fisher Information } \\
\hline & 1 & 2 & 3 & 4 & 5 & 6 & 7 & \multirow[b]{2}{*}{ 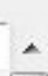 } \\
\hline 1 & $-3.7712 e+11$ & 14.8390 & 6.5110 & 6.0770 & $3.5555 \mathrm{e}+22$ & -98.1853 & 8.4515 & \\
\hline 2 & 14.8390 & 7.6782 & 4.7478 & 4.5278 & 51.3396 & -24.5165 & 4.1909 & \multirow{2}{*}{$\square$} \\
\hline 3 & 6.5110 & 4.7478 & 3.5263 & 3.4145 & 8.9707 & -8.5074 & 2.0982 & \\
\hline & \multicolumn{3}{|l|}{1} & \multicolumn{3}{|c|}{ 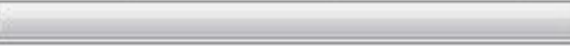 } & \multicolumn{2}{|c|}{+} \\
\hline \multicolumn{2}{|c|}{ Poles } & \multicolumn{2}{|c|}{ CRB } & \multirow{2}{*}{\multicolumn{2}{|c|}{ - Pole- Residue form }} & & & \\
\hline & 1 & & 1 & & \multirow{2}{*}{\multicolumn{2}{|c|}{+0.250000}} & & \\
\hline 1 & 1.0000 & 1 & $-1.0441 e-34$ & & & & & A \\
\hline 2 & 0.9326 & 2 & 0.0042 & & \multirow{2}{*}{\multicolumn{2}{|c|}{$(z-1.000000)$}} & & $\equiv$ \\
\hline 3 & 0.8464 & 3 & 0.2340 & & & & & \\
\hline 4 & \multirow[t]{2}{*}{0.8354} & 4 & 948.7188 & & \multicolumn{2}{|c|}{0.445897} & & \\
\hline & & & & & \multicolumn{2}{|c|}{$(7 n 0336101$} & & 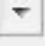 \\
\hline
\end{tabular}

Figure 4.3. Pole-residue, Fisher matrix and CRB.

FOR each node in the network

Find the $C R B$

Get the value of the bound at Fiedler eigenvalue

\section{END FOR}




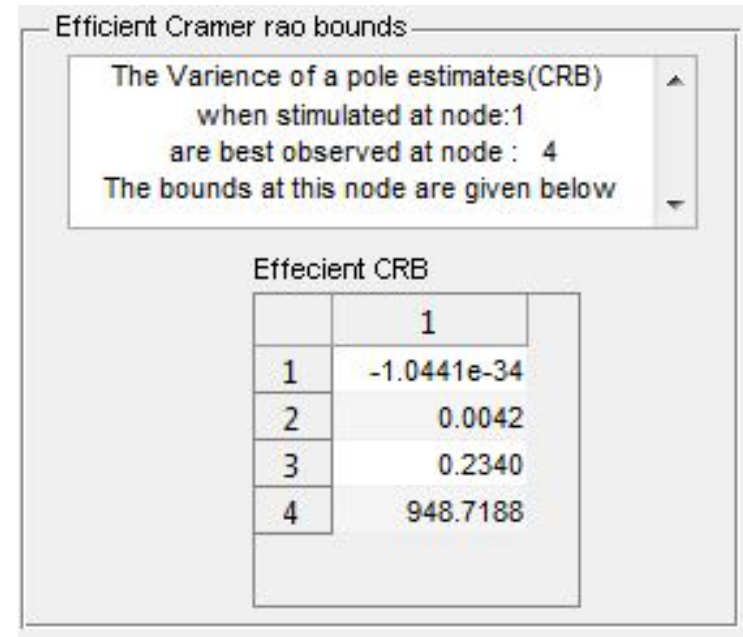

Figure 4.4. Efficient CRB

Compare all the values of the bound at Fiedler eigenvalue for smallest. Get measurement location at which the bound is small

Get the location and CRB at that measurement location 


\section{CHAPTER 5}

\section{SOME GRAPHICAL RESULTS ON ESTIMATION PERFORMANCE FOR SUBSETS OF NETWORK STRUCTURES}

The tool allows us to investigate network structure's impact on the performance of pole estimation. The graphical representation of network structure and the modification features permitted on the graph facilitate the discovery of theoretical results. In this chapter, I present some preliminary graphical results on estimation performance and their proofs.

The first theorem suggests that exchanging actuation and measurement locations does not change the estimation performance of any pole.

Theorem 5.1. Consider the estimation of an eigenvalue $\lambda_{r}$ from a network with the dynamics described in Equation (7). If the network is actuated at node $i$ and measured at node $j$, the CRB for $\lambda_{r}$ does not change if the actuation and measurement locations are switched.

Proof. According to Theorem 6 in [27], the CRB for $\lambda_{r}$ scales inversely with $\left(v_{r i} v_{r j}\right)^{2}$, where $v_{r i}$ is the ith entry in the eigenvector associated with the eigenvalue $\lambda_{r}$. After the actuation and measurement locations are switched, let us denote the new eigenvector entries associated with actuation location $j$ and measurement location $i$ as $v_{r j}^{\prime}$ and $v_{r i}^{\prime}$ respectively. The CRB now scales inversely with $\left(v_{r j}^{\prime} v_{r i}^{\prime}\right)^{2}$. As exchanging actuation and measurement locations does not change the right eigenvector of a system, I have $v_{r}=v_{r}^{\prime}$. As such, the CRB in the new setting now scales inversely with $\left(v_{r j}^{\prime} v_{r i}^{\prime}\right)^{2}=\left(v_{r j} v_{r i}\right)^{2}=\left(v_{r i} v_{r j}\right)^{2}$. The proof is complete.

In Figure 5.1, the actuation is at node 3 and the measurement is at node 1 . In Figure 5.2 , the actuation is at node 3 and the measurement is at node 3 . By comparing the outputs of two figures, I can observe that CRB's are the same even if the actuation and measurement 


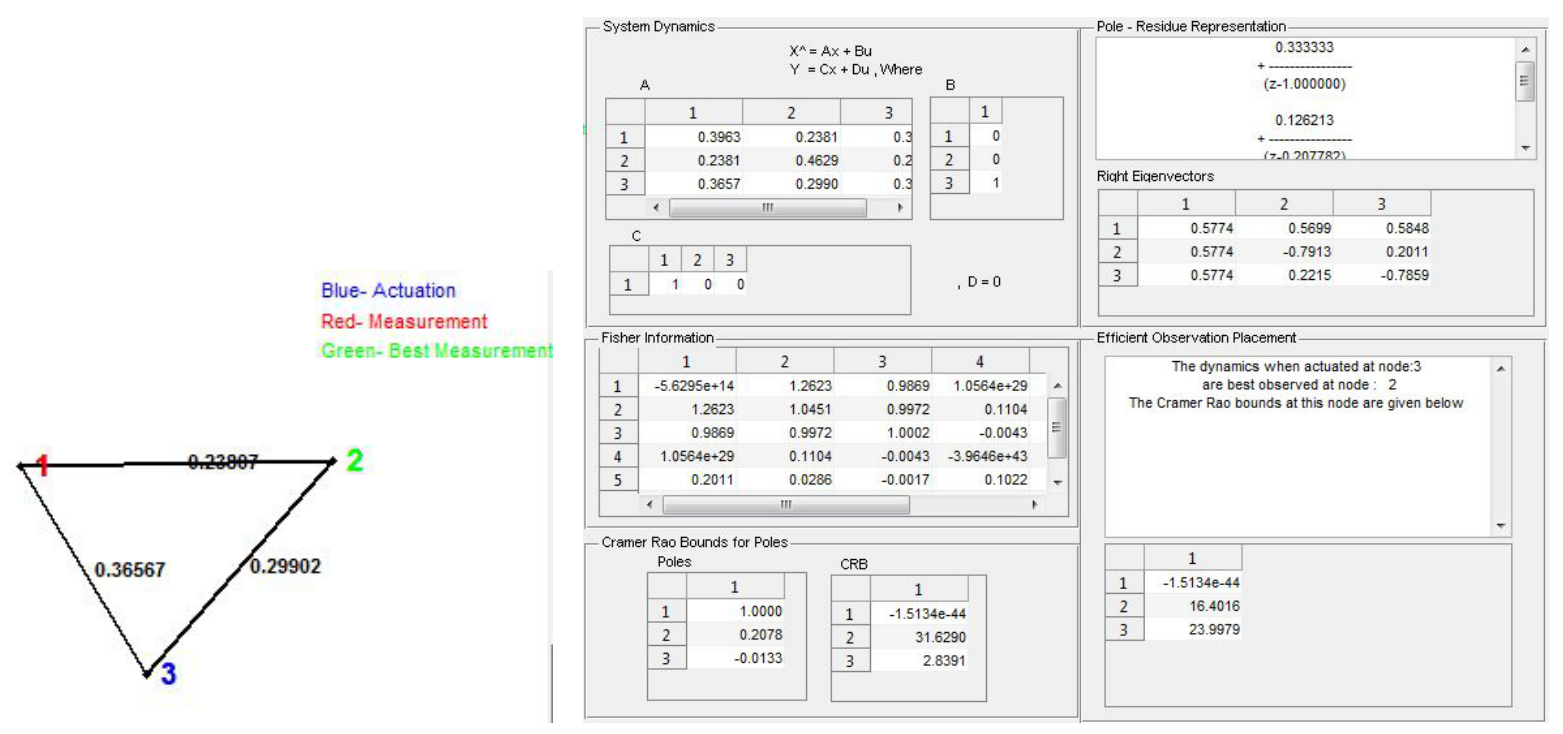

Figure 5.1. Triangle structure: actuated at node 3 and measured at node 1 (Left: Input, Right: Output).
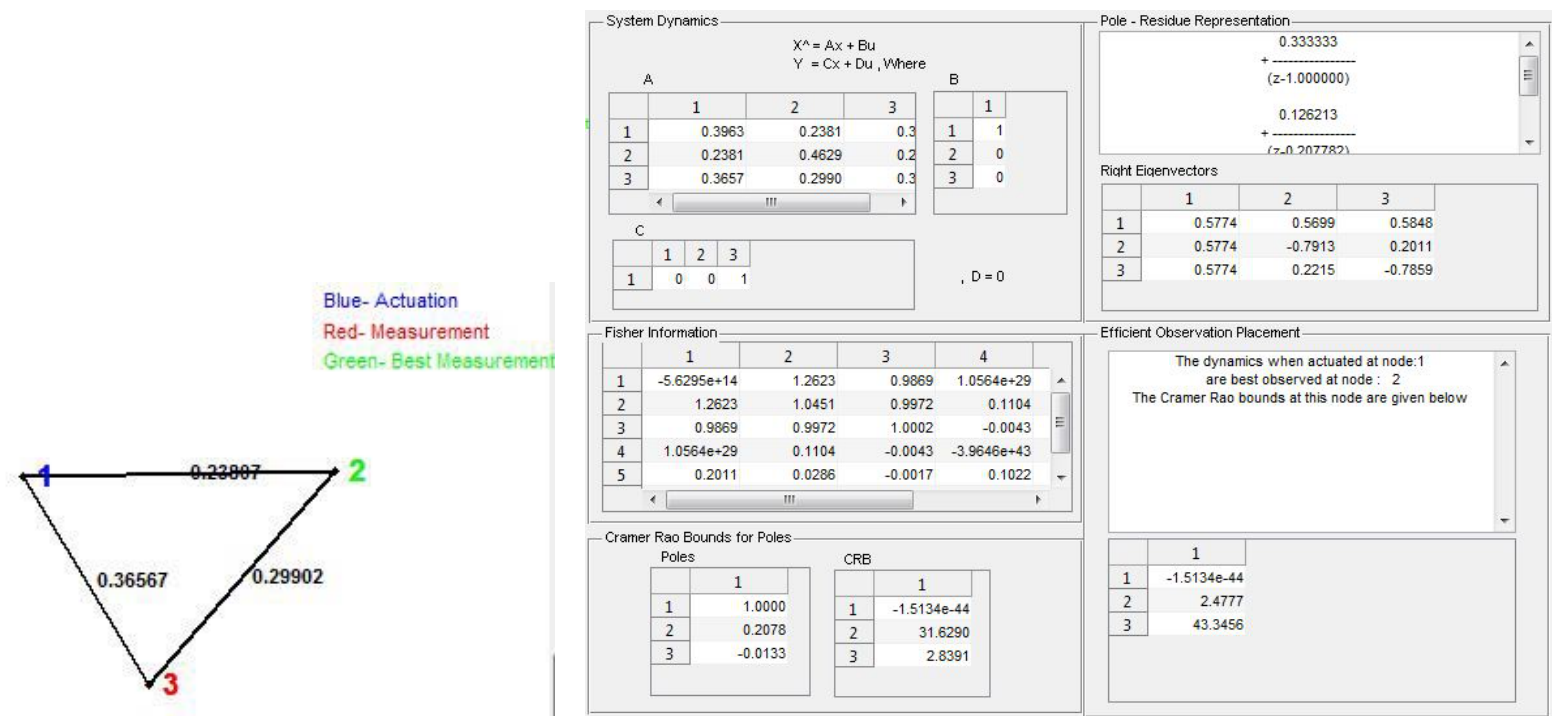

Figure 5.2. Triangle structure: actuated at node 1 and measured at node 3 (Left: Input, Right: Output).

locations are exchanged. The second theorem extends the result in [27] and informs the selection of observation locations in a tree graph when the network is actuated at a leaf of the tree. 
Theorem 5.2. Let us consider estimating the Fiedler eigenvalue of a network with a tree structure. If the network is actuated at a leaf, the minimum CRB is achieved when the measurement location is also at the leaves of the tree. Moreover, the worst observation location is among the nodes adjacent to the algebraic center of the tree.

Proof. The estimation performance of the Fiedler eigenvalue scales inversely with $\left(v_{1 i} v_{1 j}\right)^{2}$, where $i$ is the actuation location, $j$ is the measurement location, and $v_{1}$ is the eigenvector associated with the Fiedler eigenvalue, commonly called the Fiedler eigenvector. Moreover, according to $[8,7]$, entries in the Fiedler eigenvector increase or decrease monotonically from the algebraic center of the tree. As such, the maximum values of eigenvector entries are achieved at the leaves and the minimum are among the nodes adjacent to the algebraic center of the tree. Combining the above two results leads to the conclusion.

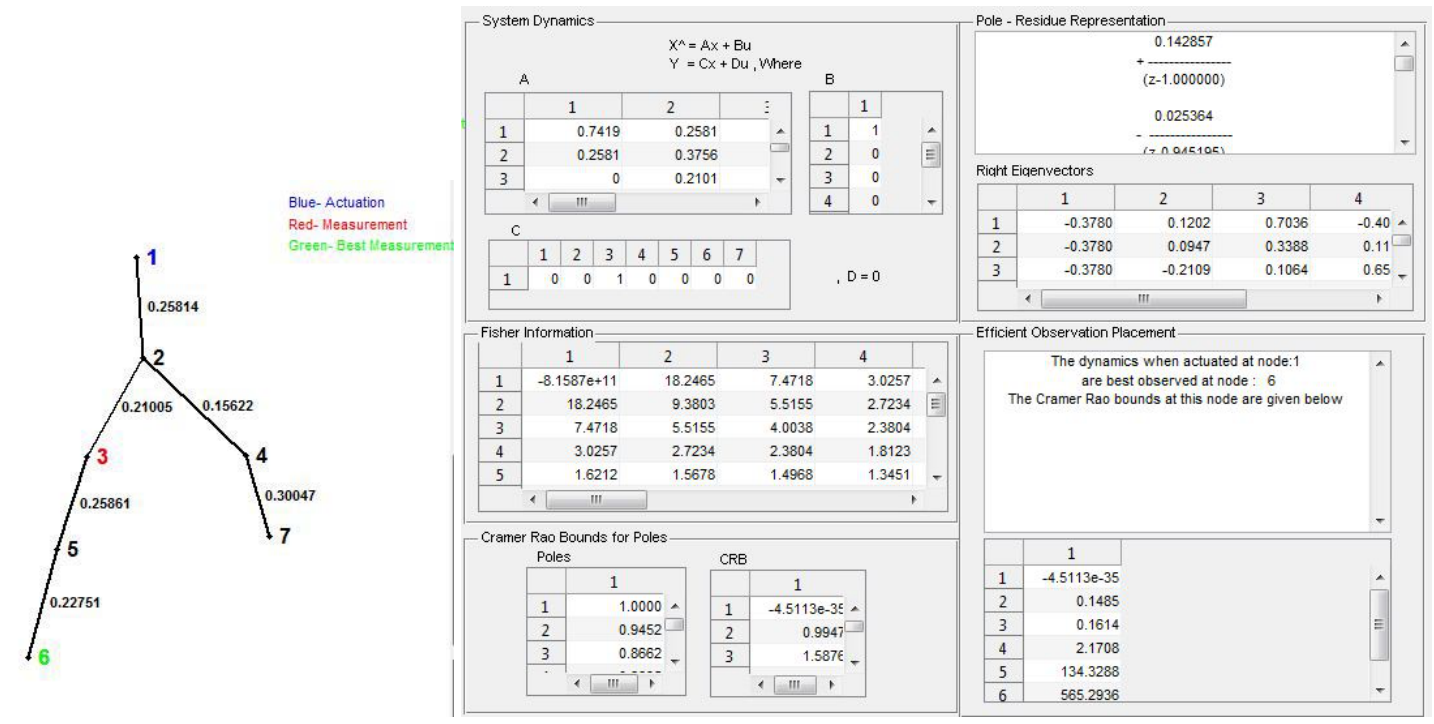

Figure 5.3. Example of input tree structure (Left: Input, Right: Output).

As seen in Figure 5.3, the actuation is placed at the leaf node 1 and measurement is at node 3. I can observe that the effective CRB(minimum CRB) is at the leaf which is at node 6. Similar characteristic holds for line structure i.e. in the line structure, the effective 
measurement location is at the end of the line, farthest from the actuation location. The example of line structure is shown in Figure 5.4.

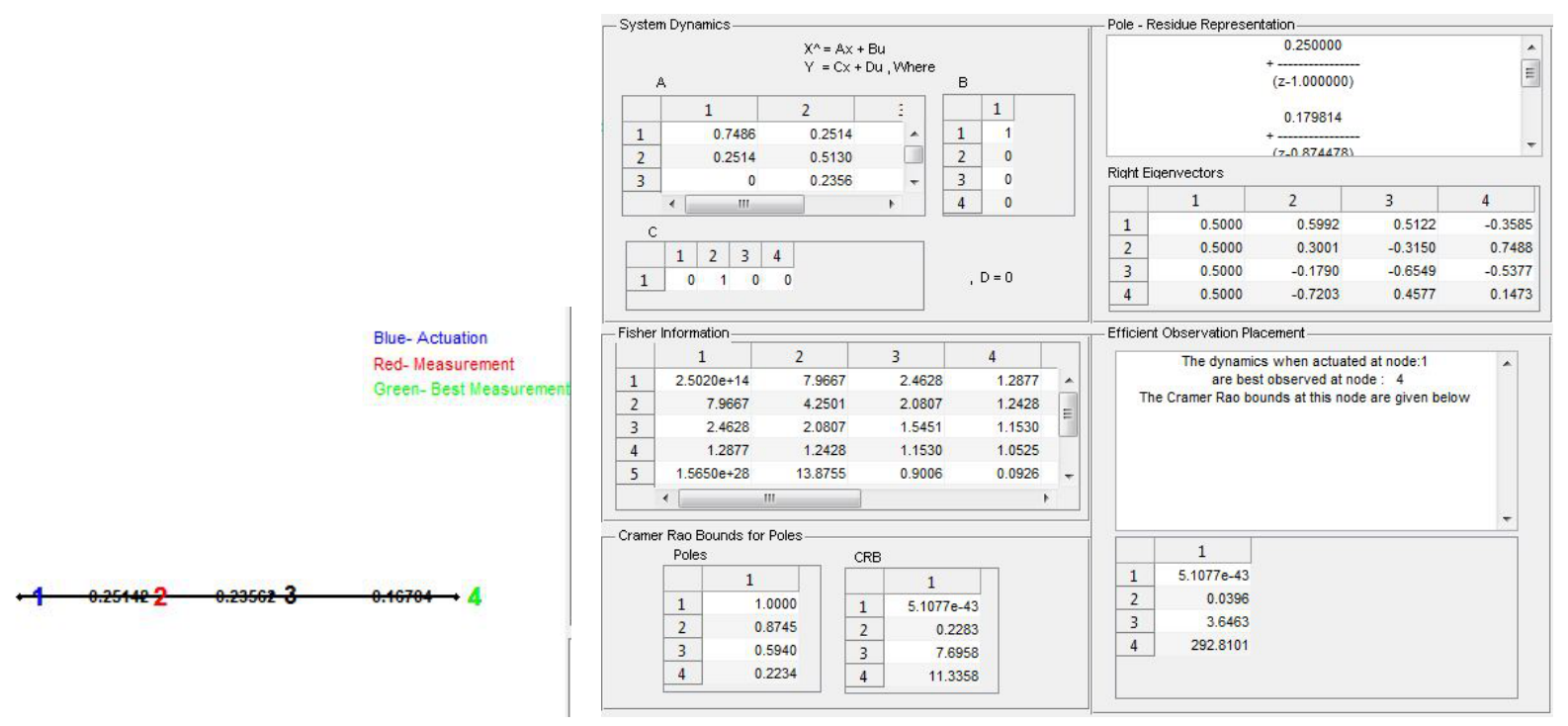

Figure 5.4. Example of line structure.

The next theorem suggests the node in a tree graph where the estimation of Fiedler eigenvalue is impossible.

Theorem 5.3. Consider the estimation of the Fiedler eigenvalue of a network with a tree structure. If the algebraic center is a node in the tree, inference is not possible if this particular node is either the actuation or measurement locations.

Proof. If the algebraic center is a node (denoted as $k$ ) in a tree, the entry $v_{1 k}$ in the Fielder eigenvector is 0 according to Theorem 4.3 in [7]. As the estimation performance of the Fiedler eigenvalue scales inversely with $\left(v_{1 i} v_{1 k}\right)^{2}$ if the network is actuated at node $i$, or $\left(v_{1 k} v_{1 j}\right)^{2}$ if the network is measured at node $j$, I obtain that the CRB is $\infty$ in each case, indicating that estimating the Fiedler eigenvalue is impossible.

The above theorem naturally leads to the conclusion that in any symmetric tree graph, 


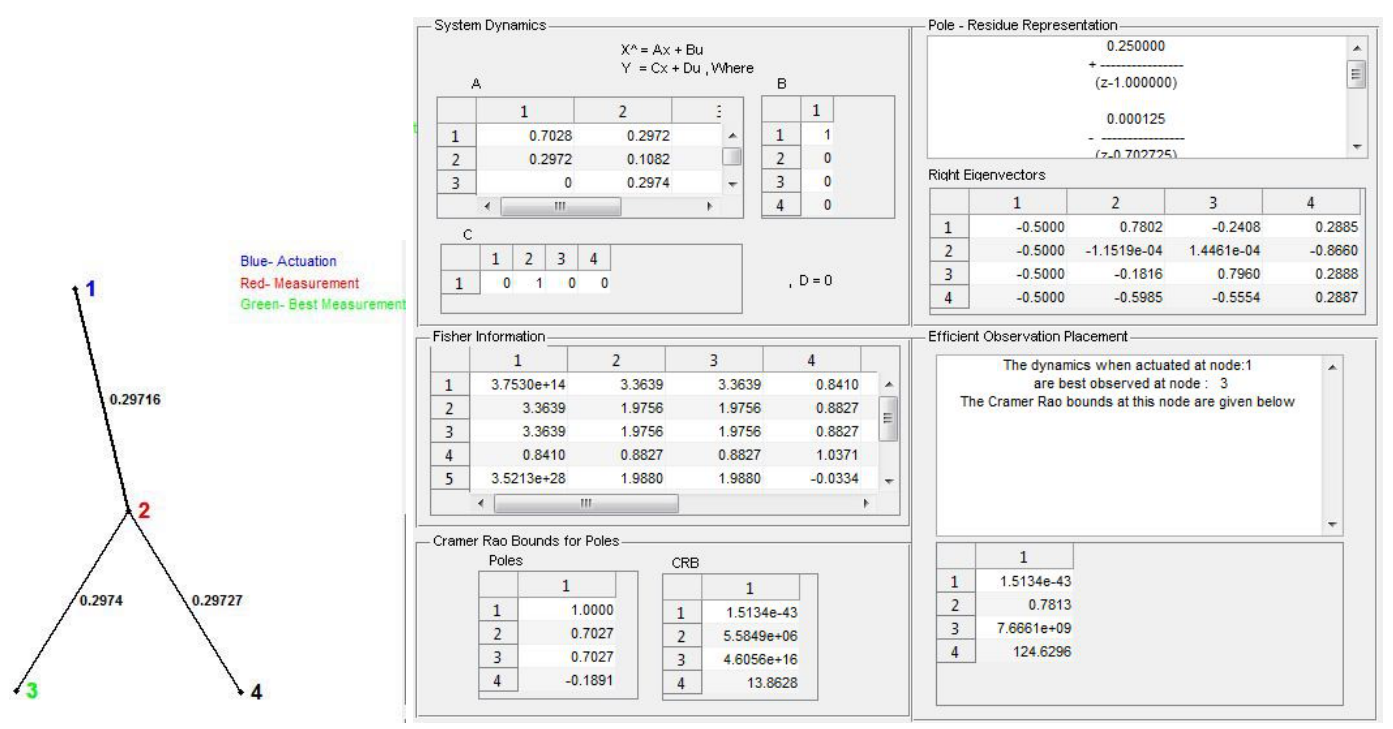

Figure 5.5. Example of symmetric tree graph.

the inference of the Fiedler eigenvalue is impossible if the network is actuated or measured at the center of the symmetry. Examples of symmetric trees include star graphs with all edge weights the same. As shown in Figure 5.5, the edge weights are nearly equal. The CRB at Fielder eigenvalue is very large or close to infinity. Therefore, inference on estimation performance is not possible.

The next theorem is concerned with the trend of CRB when the edge weight is changed in a simple graph with only two nodes.

Theorem 5.4. Consider a graph with two nodes. Increasing the edge weight between the two nodes from 0 to $\infty$ causes the CRB to increase monotonically and then decrease monotonically. The largest CRB occurs when the edge weight is 0.5 .

Proof. In the two-node case, as suggested by Equation (17), the CRB scales inversely with $\frac{\left(1+p_{1}^{2}\right)}{\left(1-p_{1}^{2}\right)^{3}}$ and $A_{1}$, where $A_{1}=\left(v_{11} v_{12}\right)^{2}$. Because the first norm of the Fielder eigenvector equals 0 , I have $v_{11}=-v_{12}$. Moreover, as $v_{11}^{2}+v_{12}^{2}=1$, I can easily obtain that $v_{11}=v_{12}=\sqrt{0.5}$, independent of the length between the two nodes. Because $A_{1}$ is a constant, $C R B$ is only 
affected by the value of the pole $p_{1}$. As the length between the two nodes increases from 0 , the Fielder eigenvalue $p_{1}$ increases from -1 to 1 , and as such causing $\frac{\left(1+p_{1}^{2}\right)}{\left(1-p_{1}^{2}\right)^{3}}$ to decrease first and then increase, with the minimum occurring while $p_{1}=0$. This occurs when the edge weight between the two nodes is 0.5 .

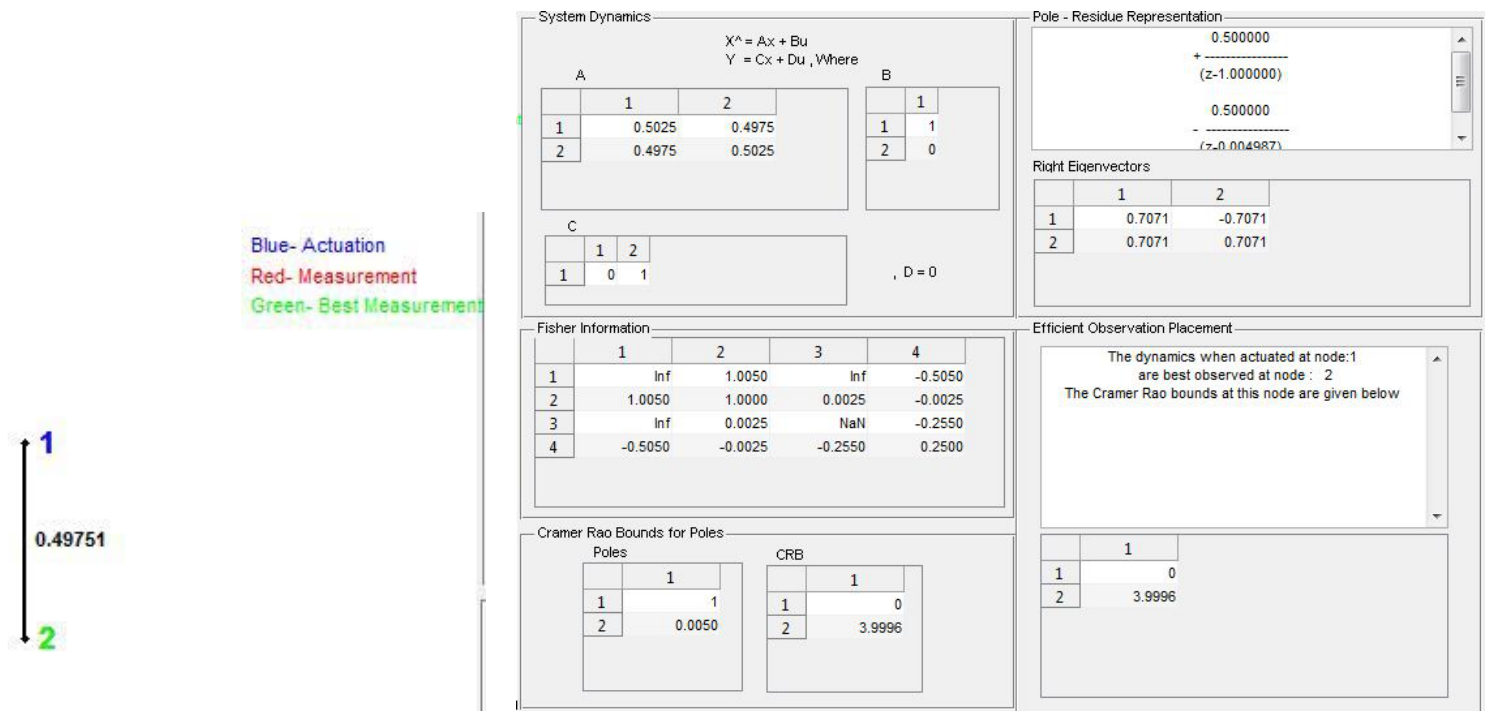

Figure 5.6. Two node graph structure.

Figure 5.6 shows the input(left) and output(right) of a two node structure when the $\mathrm{CRB}$ is maximum. The Figure 5.7 shows a graph representing that as the edge weights are increasing, the CRB increases first and then decreases. The maximum CRB is at a edge weight close to 0.5 . (The graph is plotted from the values taken from the tool by increasing edge weights for a structure shown in Figure 5.6). 


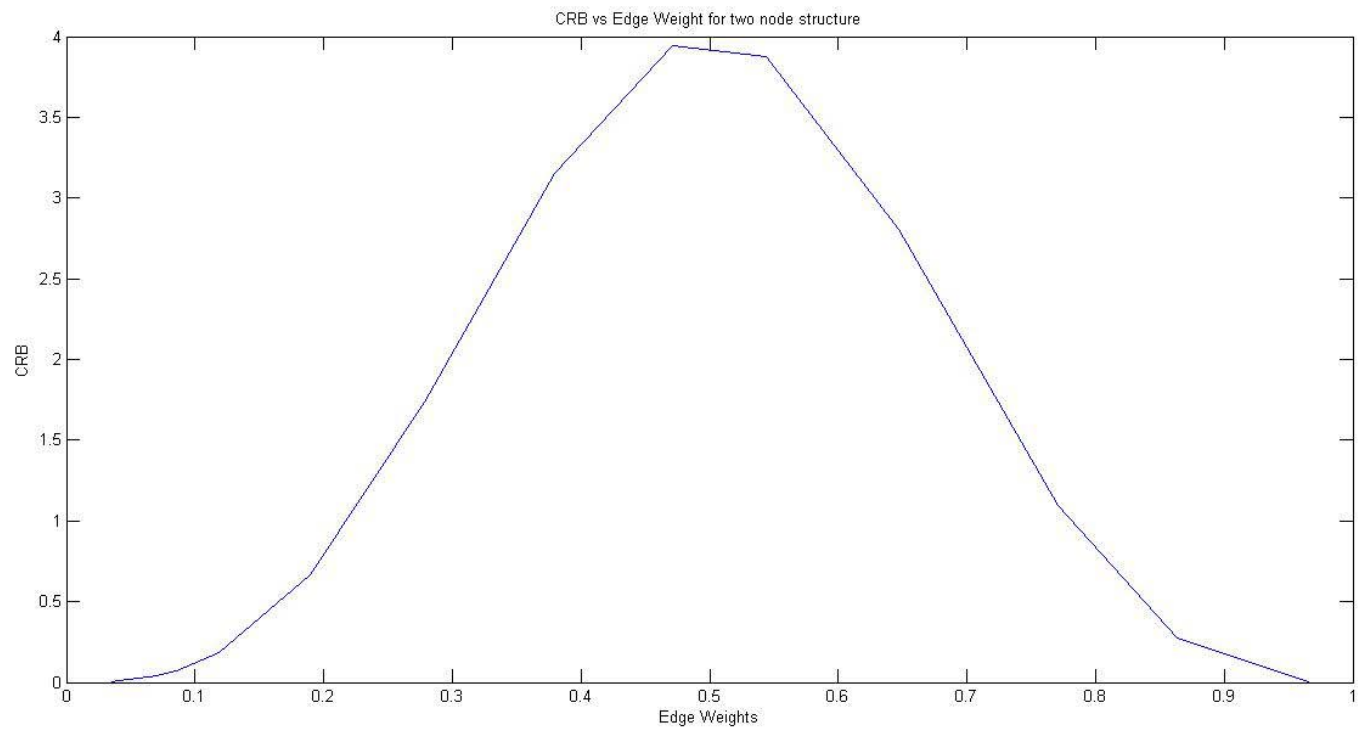

Figure 5.7. CRB vs Edge Weights for a two node graph structure. 


\section{CHAPTER 6}

\section{CONCLUDING REMARK AND FUTURE WORK}

In this thesis, I introduce an interactive tool to investigate the performance of network dynamics from noisy data. The contributions of the tool is that 1 ) it facilitates the theoretical analysis of network structure's impact on network estimation performance, and 2) it suggests the best measurement location to maximize network estimation performance, and thus provides guidance for the design of data collection experiments. In future, one can utilize the tool to develop further analytical results for non-tree structures. Moreover, I will extend the network structure from single layer to hierarchical structures, and consider network dynamics defined upon Laplacian and adjacency matrices. 
APPENDIX A

MATLAB IMPLEMANTATION OF THE TOOL USING GUIDE-GUI MAIN FUNCTION 
$1 \% \% \%$ The main function for GUI \%\%

$2 \% \%$ Developer: Veenadhar Katragadda \%\%

3 function varargout $=c r b$ (varargin)

$4 \%$ CRB MATLAB code for crb. fig

5 gui_Singleton $=1$;

6 gui_State $=$ struct('gui_Name', mfilename, ...

7 'gui_Singleton', gui_Singleton, ...

8 'gui_OpeningFcn', @erb_OpeningFcn, ...

9 'gui_OutputFcn', @erb_OutputFcn, ...

10 'gui_LayoutFen', [ ] , ..

11 'gui_Callback', []) ;

12 if nargin \&\& ischar (varargin $\{1\}$ )

13 gui_State.gui_Callback $=$ str2func(varargin $\{1\})$;

14 end

15 if nargout

16 [varargout $\{1$ :nargout $\}]=$ gui_mainfon (gui_State, varargin $\{:\})$;

17 else

18 gui_mainfon (gui_State, varargin $\{:\})$;

19 end

$20 \%$ End initialization code - DO NOT EDIT

$21 \%--$ Executes just before crb is made visible.

22 function crb_OpeningFcn (hObject, eventdata, handles, varargin) $23 \%$ This function has no output args, see OutputFcn. 
$24 \% \%$ As a opening function we declare array variables here $25 \mathrm{xy}=[] ; \% \% \mathrm{XY}$ is the $n * 2$ matrix which stores the location( $X$ and $Y$ points) of $n$ vertices.

$26 \mathrm{~A}=[] ; \% \mathrm{~A}$ is the $n * n$ matrix which has the edge definitions ( undirected or directed) between vertices

$27 \%$ rest of the variables used for intermediate calcuations

28 eid $=[]$;

$29 \operatorname{id} 1=[]$;

$30 \operatorname{xytemp}=[]$;

31 Atemp $=[]$;

32 enchk $=[]$;

33 enchk=0;

34 prev_scale $=1$;

$35 \% \%$ Updates the handles structure so that these variables can be

$36 \% \%$ used in different fucntions.

37 handles. $x y=x y$;

38 handles. A=A;

39 handles. eid=eid;

40 handles. id $1=i d 1$;

41 handles. xytemp $=$ xytemp;

42 handles. Atemp $=$ Atemp;

$43 \mathrm{handles}$. enchk=enchk; 
44 handles. prev_scale=prev_scale;

45 handles.fir_cnt $=[]$;

46 guidata (hObject, handles);

47 set (handles. enter, 'enable', ' off'); $\%$ set visibility of enter button to off

48 set (handles. undo, 'enable', 'off'); $\%$ set visibility of undo button to off

49 set (handles.uok, ' enable', ' off');

50 set (handles. check, ' enable', 'off');

51 set (handles. scale, 'enable', ' inactive');

52 set (handles. check2, 'enable', 'off' );

$53 \%$ Choose default command line output for crb

54 handles. output $=$ hObject;

$55 \%$ Update handles structure

56 guidata (hObject, handles);

$57 \%--$ Outputs from this function are returned to the command line.

58 function varargout $=$ crb_OutputFcn(hObject, eventdata, handles )

59 varargout $\{1\}=$ handles. output ;

$60 \%--$ Executes on button press in enter.

61 function enter_Callback (hObject, eventdata, handles, varargin) 62 handles. enchk=1; 
63 guidata (hObject, handles);

64 c2 $=\operatorname{get}($ handles. check2, 'value');

65 set (handles. draw, 'value', 1 ); $\%$ set the draw pop-up menu to draw mode.

66 set (handles. vertex, 'Value', 1 ); $\%$ set the vertex pop-up menu to vertex mode.

67 set (handles. check2, ' enable', 'on ') ;

68 if $c 2==0$

69 set (handles.uok, ' enable', ' off' ) ;

70 set ( handles. check, 'enable', 'on' );

71 set (handles. scale, 'enable' , 'inactive' );

72 elseif $c 2==1$

73 set (handles.uok, ' enable', ' off' );

74 set (handles. check, ' enable', 'off' ) ;

75 set (handles. scale, 'enable' , 'inactive') ;

76 end

$77 \% \%$ get the input data from the user from "input data" section

78 in_act $=$ str2double (get(handles.actuation, 'String' $)$ );

79 in_meas $=$ str2double (get (handles.measurement, 'String' $))$;

80 in_noise $=\operatorname{str}_{2}$ double (get (handles. noise, 'String' $)$ );

$81[\mathrm{~m}, \mathrm{o}]=\mathbf{s i z e}(\mathrm{h}$ andles.xy);

82 if in_act $>m$ \%if the input is higher than the number of nodes 
$83 \%$ this is the first line of the msgbox

$84 \operatorname{msgbox} T \operatorname{ext}\{1\}=$ 'You have entered a value that is higher than the number of nodes at actuation location input';

$85 \%$ this command creates the actual message box

86 msgbox(msgboxText, 'Input number incorrect', 'error') ;

87 elseif isempty ( in_act) \%if the input is not a number

$88 \%$ this is the first line of the msgbox

$89 \operatorname{msgbox} T$ ext $\{1\}=$ 'You have tried to input something that is

NOT a number at actuation location input.' ;

$90 \%$ this command creates the actual message box

91 msgbox(msgboxText, 'Input not a number', ' error') ;

92 elseif isnan( in_act) \%if the input is not a number

$93 \%$ this is the first line of the msgbox

$94 \operatorname{msgbox} T \operatorname{ext}\{1\}=$ 'Nothing is entered at actuation Iocation

input.' ;

$95 \%$ this command creates the actual message box

96 msgbox(msgboxText, 'Input empty', ' error' ) ;

97 elseif in_meas $>m \%$ if the input is higher than the number of nodes

$98 \%$ this is the first line of the msgbox

$99 \operatorname{msgbox} T \operatorname{ext}\{1\}=$ 'You have entered a value that is higher than

the number of nodes at measurement location input' ;

$100 \%$ this command creates the actual message box 
101 msgbox (msgboxText, 'Input number incorrect', ' error') ;

102 elseif isempty (in_meas) \%if the input is not a number

$103 \%$ this is the first line of the msgbox

104 msgboxText $\{1\}=$ 'You have tried to input something that is

NOT a number at measurement location input.' ;

$105 \%$ this command creates the actual message box

106 msgbox (msgboxText, 'Input not a number', 'error') ;

107 elseif isnan (in_meas) \%if the input is not a number

$108 \%$ this is the first line of the msgbox

$109 \operatorname{msgbox}$ Text $\{1\}=$ 'Nothing is entered at measurement location

input input.' ;

$110 \%$ this command creates the actual message box

111 msgbox(msgboxText, 'Input empty', ' error') ;

112 elseif isempty ( in_noise) \%if the input is not a number

$113 \%$ this is the first line of the msgbox

114 msgboxText $\{1\}=$ 'You have tried to input something that is

NOT a number at Noise varience input.';

$115 \%$ this command creates the actual message box

116 msgbox (msgboxText, 'Input not a number', ' error') ;

117 elseif isnan ( in_noise) \%if the input is not a number

$118 \%$ this is the first line of the msgbox

$119 \operatorname{msgbox}$ Text $\{1\}=$ 'Nothing is entered at Noise input.' ;

$120 \%$ this command creates the actual message box 
121 msgbox(msgboxText, 'Input empty', 'error' ) ;

122 else

$123 \% \%$ Call the matrix function to calculate several outputs for the

$124 \% \%$ current input.

$125 \mathrm{SC}=1$

126 en $=0$;

127 if $\mathrm{c} 2=1$

128 fixval = handles. fix 1 ;

129 fix $=1$;

130 else

$131 \mathbf{f i x}=0$;

132 fixval = 1 ;

133 end

134 [mat2 , p , r , F1, CRLBmat2, A1, B, C, vstr , xymid, ns, CRLBest, posi , kk, D, errchk] $=$ matrix (handles.xy, handles.A, in_act, in_meas, in_noise, sc, en, fix, fixval, handles. prev_scale);

135 handles. prev_scale $=k$;

136 guidata (hObject, handles);

137 if $\operatorname{errchk}==1$

138 errordlg ('The scaling factor cannot be possible', 'error'); 139 else

$140[\mathrm{~m} 3, \mathrm{o} 3]=\mathbf{s i z e}(\mathrm{h}$ andles. A $)$; 
141 count $=0$;

142 cla ;

143 hold on

$144 \% \%$ Display the edge weights on respective edges.

145 for $e=1: \mathrm{m} 3$

146 for $f=e+1: m 3$

147 if handles. $A(e, f)==1$

148 count $=$ count +1 ;

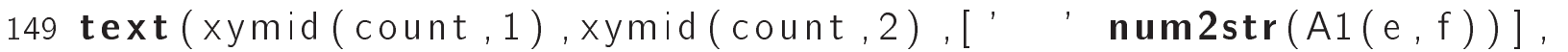

Color' ,'k', 'FontSize', 8, 'FontWeight', 'b' ) ;

150 else

151 count $=$ count $+1 ;$

152 end

153 end

154 end

$155 \% \%$ Display and plot all outputs on GUI.

156 if isempty (handles. xy)

157 plot (handles.axes, handles. xy $(:, 1)$, handles. xy $(:, 2), '$.' $)$

$158[u x, u y, d x u, d y u, d x I, d y l]=\operatorname{plotGraph}($ handles.A, handles. $x y)$;

159 n2 $=$ size (handles. A, 1);

160 plot (handles. axes, ux, uy, 'k-' ,' LineWidth' ,2)

161 plot (handles.axes, dxu, dyu, 'r:' )

162 plot (handles. axes, dxl, dyl, 'b-' ) 
$163 \%$ plot (handles.axes, ix, iy, 'ko')

164 plot (handles.axes, handles.xy $(:, 1)$, handles.xy $(:, 2)$, 'k.' $)$

165 for $k=1: n 2$

166 text (handles. xy $(k, 1)$, handles.xy $(k, 2)$, [' ' num2str(k) ], 'Color'

$$
\text { , 'k', 'FontSize', 12, 'FontWeight ', 'b ') }
$$

167 end

168 end

169 text (handles. xy (in_act, 1 ), handles. xy (in_act, 2), [', ' num 2str( in_act)], 'Color' , 'b ', 'FontSize' ,12, 'FontWeight' , 'b ')

170 text (handles.xy (in_meas, 1), handles.xy (in_meas, 2), [' ' num2str (in_meas)], 'Color', 'r', 'FontSize',12, 'FontWeight', 'b ') 171 text (handles.xy (posi, 1), handles.xy (posi, 2), [', ', num2str(posi) ] , 'Color' , 'g' , 'FontSize ', 12, 'FontWeight ', 'b ')

172 text (7,9.15, 'Blue- Actuation', 'Color' ,'b' , 'FontSize', 8)

173 text (7,8.83, 'Red- Measurement', 'Color' , 'r' , 'FontSize', 8)

174 text $(7,8.51$, 'Green- Best Measurement ', 'Color' , 'g' , 'FontSize' ,8)

175 if length $(d x \mid)>=2$

176 if $\quad$ isnan $(d x I(1,1)+d x I(2,1))$

177 text $(0.52,9.47$, 'Directed edges: ', 'FontSize', 8) 178 text $(0.52,9.15$, ,-- Blue large to small node', 'Color', 'b', ' FontSize', 8) 
179 text $(0.52,8.83$, '-- Red small to large node', 'Color', 'r', '

FontSize', 8)

180 else

181 end

182 else

183 end

184 if length $(\mathrm{d} x u)>=2$

185 if isnan $(\mathrm{d} \times u(1,1)+\mathrm{d} \times \mathrm{u}(2,1))$

186 text (0.52,9.47, 'Directed edges:' , 'FontSize', 8)

187 text $(0.52,9.15$, '- Blue large to small node', 'Color', 'b', '

FontSize', 8)

188 text $(0.52,8.83$, '-- Red small to large node', 'Color', 'r', ' FontSize', 8 )

189 else

190 end

191 else

192 end

193 set (handles. scale, 'Units', 'normalized', 'String', kk);

194 set (handles.rev, 'Units', 'normalized', 'Data', D) ;

195 set (handles. poles, 'Units', 'normalized', 'Data', p);

196 set (handles. fisher, 'Units', 'normalized', 'Data', F1);

197 set (handles.crb, 'Units', 'normalized', 'Data', CRLBmat2);

198 set(handles.a, 'Units', 'normalized', 'Data', A1); 


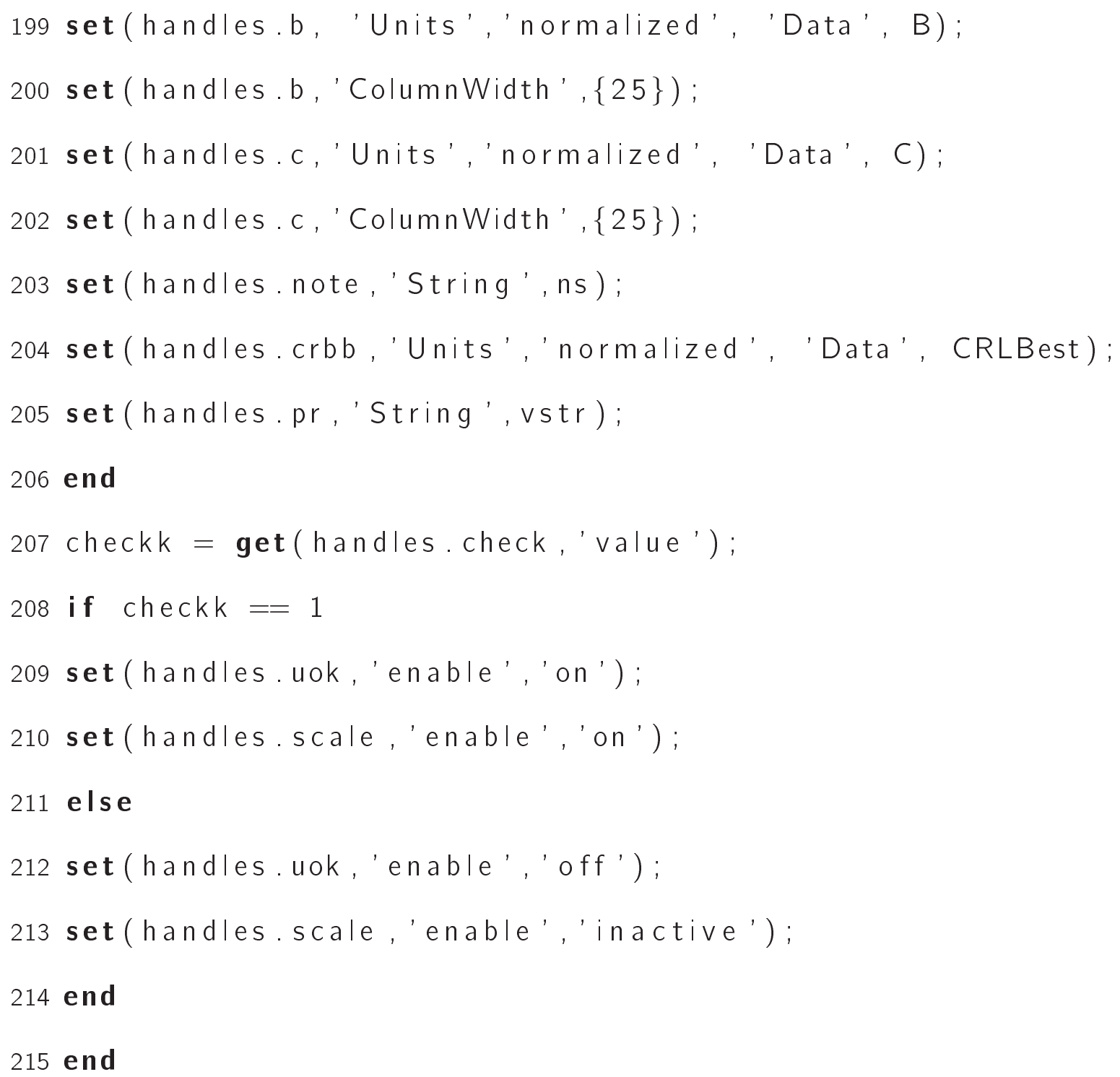


221 set (handles. vertex, 'Value', 1); $\% \%$ Set the vertex pop up menu to vertex mode.

222 set (handles.draw, 'Value', 1 ); $\%$ Set the draw popup menu to draw mode.

223 else

$224 \%$ Make the variables empty if they are not.

$225 \times \mathrm{y}=[]$

$226 \mathrm{~A}=[]$;

227 eid $=[]$;

228 id $1=[]$;

229 xytemp $=[]$;

230 Atemp $=[]$;

231 enchk =[];

232 enchk =0;

233 handles. $x y=x y$;

234 handles. $A=A$;

235 handles . eid=eid;

236 handles. id $1=i d 1$;

237 handles. xytemp $=$ xytemp ;

238 handles. Atemp $=$ Atemp;

239 handles. enchk=enchk;

240 guidata (hObject, handles);

241 end 
242 case $2 \%$ Open saved netwrok.

243 if isempty(handles. xy)

$244 \% \%$ Create a dialogue box.

245 ans $=$ questdlg('Are you sure you want to open a saved graph?

This operation cannot be undone. ', ...

246 'Open a Graph', ...

247 'Yes', , 'No', 'No' );

248 switch ans

249 case 'Yes' \% Open a user interface box to select to open the saved network.

$250 \mathrm{~A}=[]$;

$251 \times y=[]$;

252 uiopen('*.mat'); \% open a user interface box.

253 handles. $A=A$;

254 handles. $x y=x y$;

255 guidata (hObject, handles);

256 if isempty (handles. $x y)$

257 hold on

258 cla; \% clear the axes

$259 \%$ Plot the saved network on the axes of GUI.

260 if isempty(handles. $x y)$

261 plot (handles.axes, handles. xy $(:, 1)$, handles. xy $(:, 2), '$.')

$262[u x, u y, d x u, d y u, d x l, d y l]=\operatorname{plotGraph}($ handles. A, handles. $x y)$; 
$263 n 2=\operatorname{size}($ handles. A, 1$)$;

264 plot (handles. axes, ux, uy, 'k-' ,' LineWidth' ,2)

265 plot (handles. axes, dxu, dyu, ' $r:{ }^{\prime}$ )

266 plot (handles. axes, dxl, dyl , 'b--' )

$267 \%$ plot (handles.axes, ix, iy, 'ko')

268 plot (handles. axes, handles. xy $(:, 1)$, handles. $x y(:, 2)$, 'k. ')

269 for $k=1: n 2$

270 text (handles. xy $(k, 1)$, handles.xy $(k, 2)$, [', ' num2str $(k)]$, ' Color' , 'k', 'FontSize',12, 'FontWeight', 'b ')

271 end

272 end

$273 \% \%$ Set motion function to off and make it on to avoid the delay of

$274 \% \%$ the function

275 set (gcf, 'WIndowButtonMotionFcn', ' ' ) ;

276 set (gcf, 'WIndowButtonMotionFcn', \{

(afigure1_WindowButtonMotion Fcn, handles \});

277 set (handles. enter, ' enable', 'on'); $\%$ Set visibility of enter button to on

278 set (handles. undo, 'enable', 'off'); $\%$ Set visibility of undo button to off

279 set (handles. save, 'value', 1 ); $\%$ Set the save pop menu to "plot a network" option. 
280 set (handles. new, ' enable', 'on ') ;

281 else

282 set (handles. enter, 'enable', 'off'); $\%$ Set visibility of enter button to off

283 set (handles. undo, 'enable', 'off'); $\%$ Set visibility of undo button to off

284 set (handles. save, 'value', 1 ); $\% \%$ Set the save pop menu to "plot a network" option.

285 end

286 otherwise

287 set (handles. save, 'value', 1 ); $\%$ Set the save pop menu to "plot a network" option.

288 end

289 else

$290 \mathrm{~A}=[]$

$291 \times y=[]$

292 uiopen ('*.mat');

293 handles. $A=A$;

294 handles. $x y=x y$;

295 guidata (hObject, handles);

296 if isempty (handles. xy)

297 hold on

298 cla ; 
if isempty (handles. xy)

300 plot (handles. axes, handles. xy $(:, 1)$, handles. xy $(:, 2)$, . ' $)$

$301[u x, u y, d x u, d y u, d x l, d y l]=\operatorname{plotGraph}($ handles.A, handles. $x y)$;

$302 \mathrm{n} 2=\operatorname{size}($ handles. $\mathrm{A}, 1)$;

303 plot (handles. axes, ux, uy, 'k-' , ' LineWidth ' ,2)

304 plot (handles.axes, dxu, dyu, 'r: ')

305 plot (handles.axes, $d x \mid, d y l, ' b-'$ )

$306 \%$ plot (handles.axes, ix, iy, 'ko')

307 plot (handles. axes, handles. xy $(:, 1)$, handles. xy $(:, 2)$, 'k. ')

308 for $k=1: n 2$

309 text (handles. xy $(k, 1)$, handles. xy $(k, 2)$, [', ' num2str $(k)]$, 'Color' , 'k', 'FontSize', 12 , 'FontWeight' , 'b' )

310 end

311 end

312 set (gcf, 'WIndowButtonMotionFcn', ', ') ;

313 set ( gcf, 'WIndowButtonMotionFcn', \{

(ofigure1_WindowButtonMotionFcn, handles \}) ;

314 set (handles. enter, 'enable' , 'on' );

315 set (handles. undo, ' enable', 'on ') ;

316 set ( handles. save, 'value ', 1 ) ;

317 set (handles. new, ' enable', 'on ') ;

318 else

319 set (handles. enter, ' enable ', 'off' ); 


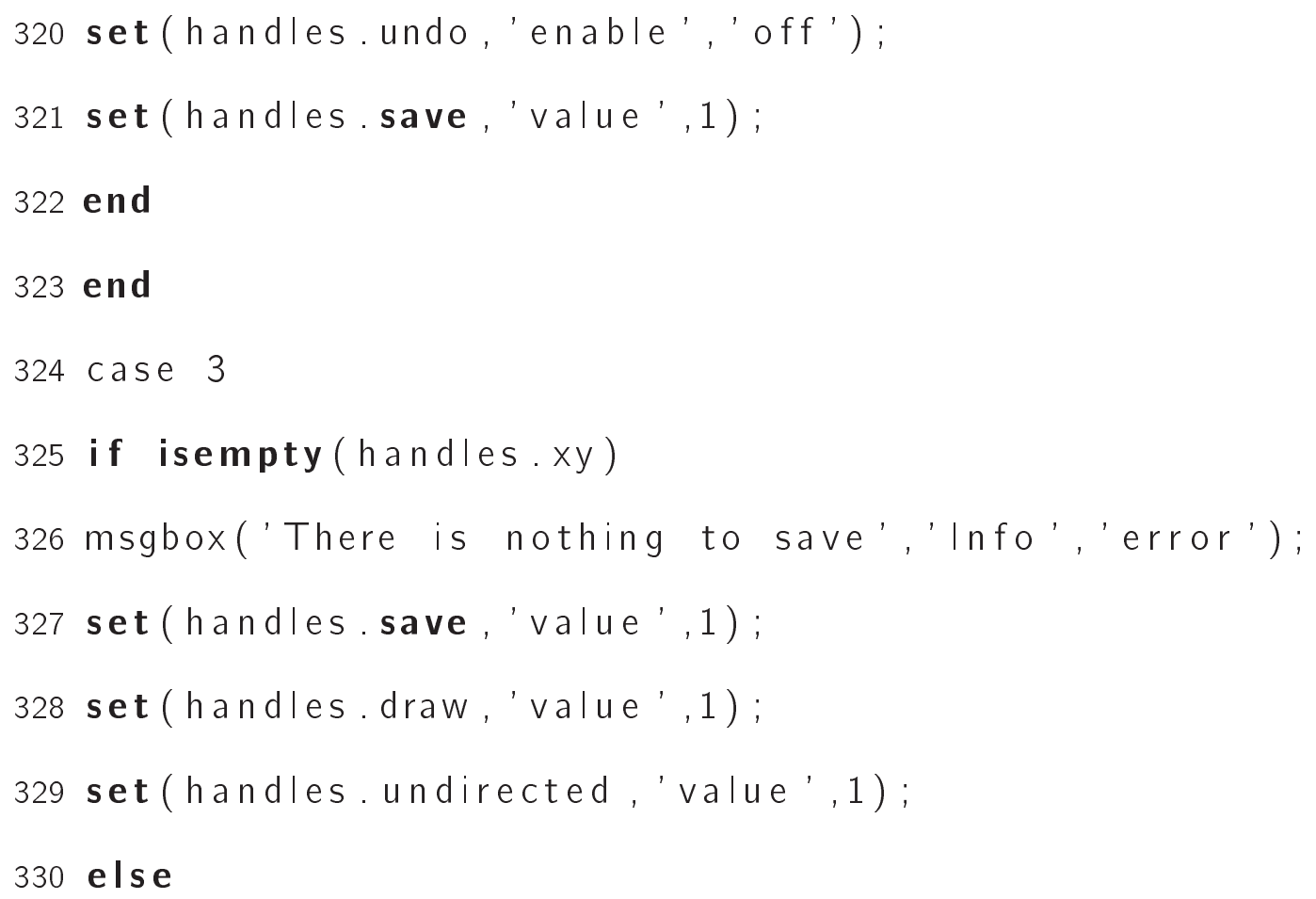


$343 \vee=\operatorname{get}($ handles.draw, 'value' );

$344 \vee 1=\operatorname{get}($ handles. vertex, 'value' );

345 if $v==3$

346 set ( handles. vertex, 'value', 1 );

347 msgbox( 'Cannot use edge mode', 'Info', ' error' );

348 end

349 if $\vee 1==2$

350 if isempty (handles. xy)

351 msgbox('Cannot draw/delete edges without Vertices/Nodes', '

Error in GUI', 'error');

352 set ( handles. vertex, 'value', 1 );

353 end

354 else

355 end

$356 \%--$ Executes on selection change in draw.

357 function draw_Callback(hObject, eventdata, handles)

$358 \mathrm{v}=\operatorname{get}($ handles.draw, 'value');

$359 \vee 1=\operatorname{get}($ handles. vertex, 'value' ) ;

360 if $\quad \mathrm{v}==2$

361 if isempty (handles. $x y$ )

362 msgbox('Cannot delete Vertices/Edges without Nodes', 'Error in

$$
\text { GUI', ' error') ; }
$$

363 set (handles. draw, 'value', 1 ); 


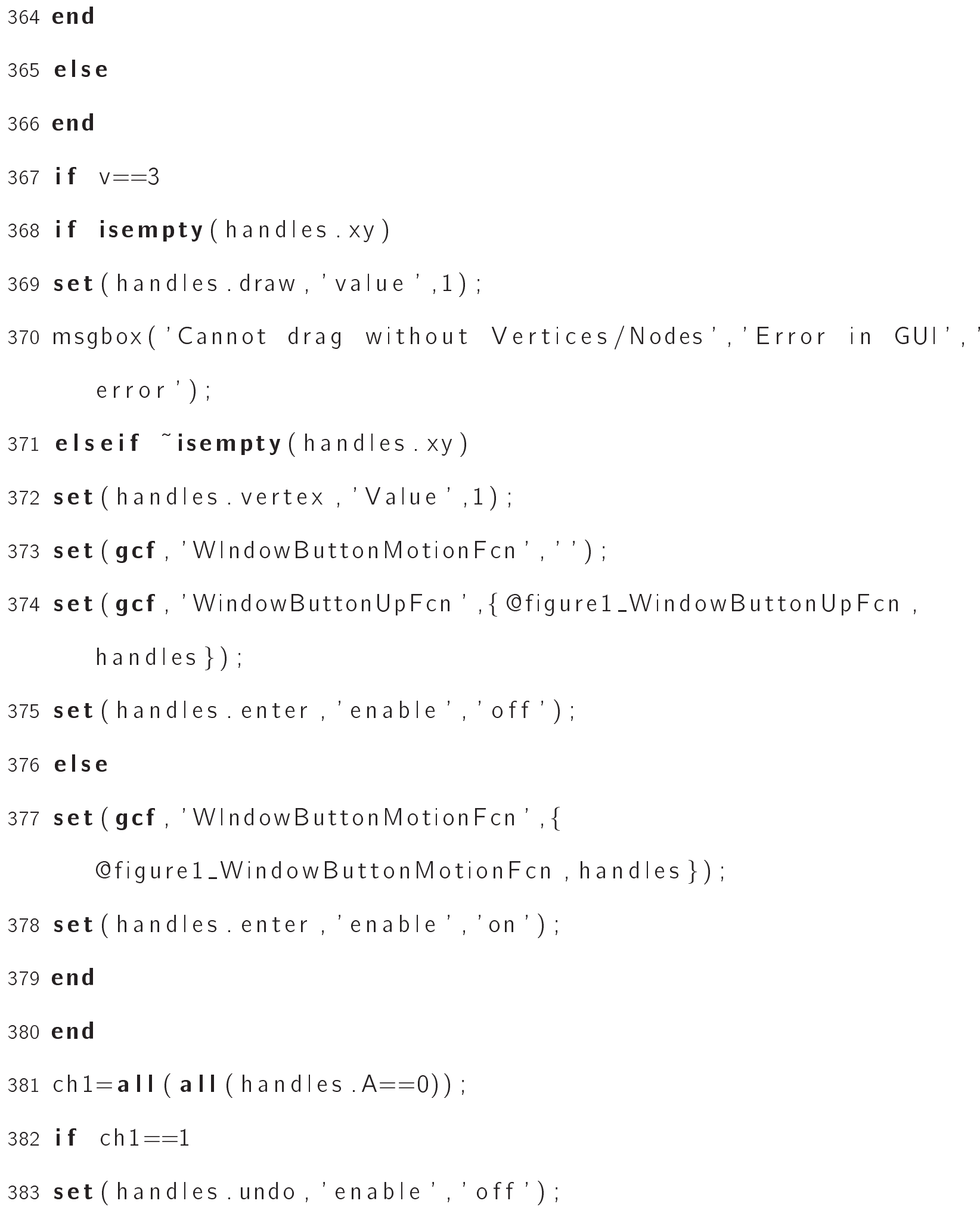


384 end

385 function figure1_WindowButtonMotionFcn (hObject, eventdata, handles )

386 clc ;

387 axX $=$ axis ;

388 pttt=getPoint ;

389 if $(\operatorname{pttt}(1,1)<\operatorname{axx}(1,1))$

390 set ( handles. xytext, 'String', ' ' ) ;

391 elseif $(\operatorname{pttt}(1,1)>\operatorname{axx}(1,2))$

392 set ( handles. xytext, 'String ', ' ') ;

393 elseif $(\operatorname{pttt}(1,2)<\operatorname{axx}(1,3))$

394 set (handles. xytext, 'String', ' ') ;

395 elseif $(\operatorname{pttt}(1,2)>\operatorname{axx}(1,4))$

396 set ( handles. xytext, 'String ', ' ') ;

397 else

$398 \vee 3=\operatorname{get}($ handles.draw, 'value' ) ;

$399 \vee 4=\operatorname{get}($ handles. vertex, 'value' );

$400 \vee 5=\operatorname{get}($ handles. undirected, 'value') ;

$401 \mathrm{pt}=$ getPoint ;

402 if $\vee 3==1 \quad \& \& \quad \vee 4==1$

403 set (handles. xytext, ' String ', $\left[{ }^{\prime} x={ }^{\prime}\right.$ num2str(pt) ' $=y$ ' $\left.^{\prime}\right)$;

404 elseif isempty (handles. $x y$ )

405 id $=$ get NearestVertex (pt, handles. $x y)$; 


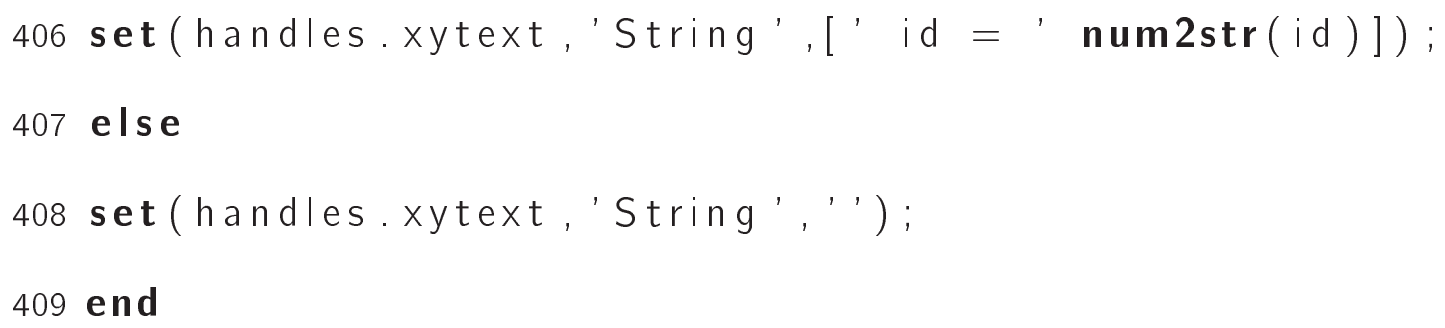


430 plot (handles. axes, $d x l, d y l, ' b--'$ )

$431 \%$ plot (handles. axes, ix, iy, 'ko')

432 plot (handles.axes, handles.xy $(:, 1)$, handles. xy $(:, 2)$, 'k.' )

433 for $k=1: n 2$

434 text ( handles. xy $(k, 1)$, handles. xy $(k, 2)$, [' num2str $(k)]$, ' Color' , 'k', 'FontSize ', 12 , 'FontWeight' , 'b ')

435 end

436 end

437 elseif $\mathrm{ch}==0$

438 cla ;

439 hold on

440 if isempty (handles. xy)

441 plot (handles. axes, handles. xy $(:, 1)$, handles. xy $(:, 2), '$.' $)$

$442[u x, u y, d x u, d y u, d x l, d y l]=$ plotGraph (handles.A, handles. $x y, a x)$;

443 n2 $=\operatorname{size}($ handles. A, 1);

444 plot (handles. axes, ux, uy, 'k-' ,' LineWidth' ,2)

445 plot (handles. axes, dxu, dyu, 'r: ')

446 plot (handles. axes, $d x l, d y l, ' b-'$ )

$447 \%$ plot (handles. axes, ix, iy, 'ko')

448 plot (handles.axes, handles. xy $(:, 1)$, handles. xy $(:, 2)$, ' $k$.' ')

449 for $k=1: n 2$ 
450 text (handles. xy $(k, 1)$, handles. xy $(k, 2)$, [', ' num2str $(k)]$, ' Color' , 'k', 'FontSize ', 12, 'FontWeight' , 'b' )

451 end

452 in_act $=$ str2double (get(handles.actuation, 'String') );

453 in_meas $=$ str2double (get (handles.measurement, 'String ') );

454 in_noise $=\operatorname{str}_{2}$ double (get (handles. noise, 'String ')) ;

$455[\mathrm{~m}, \mathrm{o}]=\mathbf{s i z e}($ handles.xy);

456 if in_act $>m$ \%if the input is higher than the number of nodes $457 \%$ this is the first line of the msgbox

458 msgboxText $\{1\}=$ 'You have entered a value that is higher than the number of nodes at actuation location input' ;

$459 \%$ this command creates the actual message box

460 msgbox (msgboxText, 'Input number incorrect', 'error') ;

461 elseif isempty (in_act) \%if the input is not a number

$462 \%$ this is the first line of the msgbox

$463 \operatorname{msgbox} T$ ext $\{1\}=$ 'You have tried to input something that is

NOT a number at Noise varience input at actuation location input.' ;

$464 \%$ this command creates the actual message box

465 msgbox(msgboxText, 'Input not a number', 'error') ;

466 elseif isnan(in_act) \%if the input is not a number

$467 \%$ this is the first line of the msgbox 
468 msgboxText $\{1\}=$ 'Nothing is entered at actuation location input.' ;

$469 \%$ this command creates the actual message box

470 msgbox(msgboxText, 'Input empty', ' error') ;

471 elseif in_meas $>m$ \%if the input is higher than the number of nodes

$472 \%$ this is the first line of the msgbox

473 msgboxText $\{1\}=$ 'You have entered a value that is higher than the number of nodes at measurement location input' ;

$474 \%$ this command creates the actual message box

475 msgbox (msgboxText, 'Input number incorrect', ' error');

476 elseif isempty (in_meas) \%if the input is not a number

$477 \%$ this is the first line of the msgbox

478 msgboxText $\{1\}=$ 'You have tried to input something that is

NOT a number at Noise varience input at measurement

location input.' ;

$479 \%$ this command creates the actual message box

480 msgbox(msgboxText, 'Input not a number', ' error') ;

481 elseif isnan (in_meas) \%if the input is not a number

$482 \%$ this is the first line of the msgbox

$483 \operatorname{msgbox} T$ ext $\{1\}=$ 'Nothing is entered at measurement location

input input.' ;

$484 \%$ this command creates the actual message box 
485 msgbox (msgboxText, 'Input empty', 'error') ;
486 elseif isempty (in_noise) \%if the input is not a number

$487 \%$ this is the first line of the msgbox

488 msgboxText $\{1\}=$ 'You have tried to input something that is

NOT a number at Noise varience input.';

$489 \%$ this command creates the actual message box

490 msgbox (msgboxText, 'Input not a number', ' error' ) ;

491 elseif isnan ( in_noise) \%if the input is not a number

$492 \%$ this is the first line of the msgbox

493 msgboxText $\{1\}=$ 'Nothing is entered at Noise input.' ;

$494 \%$ this command creates the actual message box

495 msgbox(msgboxText, 'Input empty', ' error' ) ;

496 else

497 S C $=1$;

498 en $=0$;

499 c2 $=$ get (handles. check2, 'value');

500 if $\quad$ C2 $=1$

501 fixval = handles. fix 1 ;

502 fix $=1$;

503 else

$504 \mathbf{f i x}=0$;

$505 \mathrm{fixval}=1$;

506 end 
507 [mat2 , p , r , F1, CRLBmat2, A1, B, C, vstr , xymid, ns, CRLBest, posi , kk, D, errchk] = matrix (handles. xy, handles.A, in_act, in_meas, in_noise, sc, en, fix, fixval, handles.prev_scale);

508 if handles.prev_scale $\sim$ = kk

509 set (gcf, 'WIndowButtonMotionFcn', ', ') ;

510 set (gcf, 'WIndowButtonMotionFcn', \{

(Qfigure1_WindowButtonMotionFcn, handles \}) ;

511 end

512 handles. prev_scale $=$ kk;

513 guidata (hObject, handles);

514 set (gcf, 'WIndowButtonMotionFcn', \{

(ofigure1_WindowButtonMotion Fcn, handles \}) ;

515 if $\operatorname{errchk}==1$

516 set (gcf, 'WIndowButtonMotionFcn', ', ') ;

517 errordlg ('Further dragging cannot be possible beacause of scaling constraint', 'error');

518 else

$519[\mathrm{~m} 3, \mathrm{o} 3]=\mathbf{s i z e}(\mathrm{h}$ andles. A ) ;

520 count $=0$;

521 for $e=1: m 3$

522 for $f=e+1: m 3$

523 if handles. $A(e, f)==1$

524 count $=$ count $+1 ;$ 
525 text (xymid (count, 1 ) , xymid (count , 2) , [', ' num 2str(A1(e,f)) ], '

Color' , 'k' , 'FontSize', 8, 'FontWeight' , 'b' );

526 else

527 count $=$ count +1

528 end

529 end

530 end

531 hold on

532 text (handles. xy (in_act, 1 ), handles. xy (in_act, 2), [', 'num2str(

in_act)] , 'Color', 'b ', 'FontSize' '12, 'FontWeight' , 'b ')

533 text (handles.xy (in_meas, 1), handles.xy(in_meas, 2), [' ' num2str

(in_meas) ], 'Color', 'r' , 'FontSize',12, 'FontWeight ', 'b ')

534 text (handles.xy (posi, 1), handles.xy (posi, 2), [' ', num2str(posi)

], 'Color', 'g', ' FontSize', 12, 'FontWeight' , 'b')

535 text (7,9.15,'Blue- Actuation', 'Color' ,'b' , 'FontSize', 8)

536 text $(7,8.83$, 'Red- Measurement' , 'Color', 'r' , 'FontSize', 8)

537 text $(7,8.51$, 'Green- Best Measurement ', 'Color' , 'g', 'FontSize'

,8)

538 if length $(d x \mid)>=2$

539 if isnan $(\mathrm{d} \times \mathrm{I}(1,1)+\mathrm{d} \times \mathrm{I}(2,1))$

540 text $(0.52,9.47$, ' Directed edges: ', 'FontSize', 8$)$

541 text $(0.52,9.15$, '-- Blue large to small node', 'Color', 'b' ,'

FontSize', 8) 
542 text $(0.52,8.83$, ,-- Red small to large node', 'Color', 'r', '

FontSize', 8 )

543 else

544 end

545 else

546 end

547 if length $(\mathrm{d} \times \mathrm{u})>=2$

548 if isnan $(\mathrm{d} \times u(1,1)+\mathrm{d} \times \mathrm{u}(2,1))$

549 text $(0.52,9.47$, 'Directed edges:' ,' 'FontSize', 8)

550 text $(0.52,9.15$, '-- Blue large to small node', 'Color', 'b', '

FontSize', 8)

551 text $(0.52,8.83$, '-- Red small to large node', 'Color', 'r', '

FontSize', 8)

552 else

553 end

554 else

555 end

556 set (handles. scale, 'Units', 'normalized', 'String', kk);

557 set (handles.rev, 'Units', 'normalized', 'Data', D);

558 set (handles.poles, 'Units', 'normalized', 'Data', p);

559 set (handles. fisher, 'Units', ' normalized', 'Data', F1);

560 set (handles.crb, 'Units', 'normalized', 'Data', CRLBmat2);

561 set (handles.a, 'Units', 'normalized', 'Data', A1); 


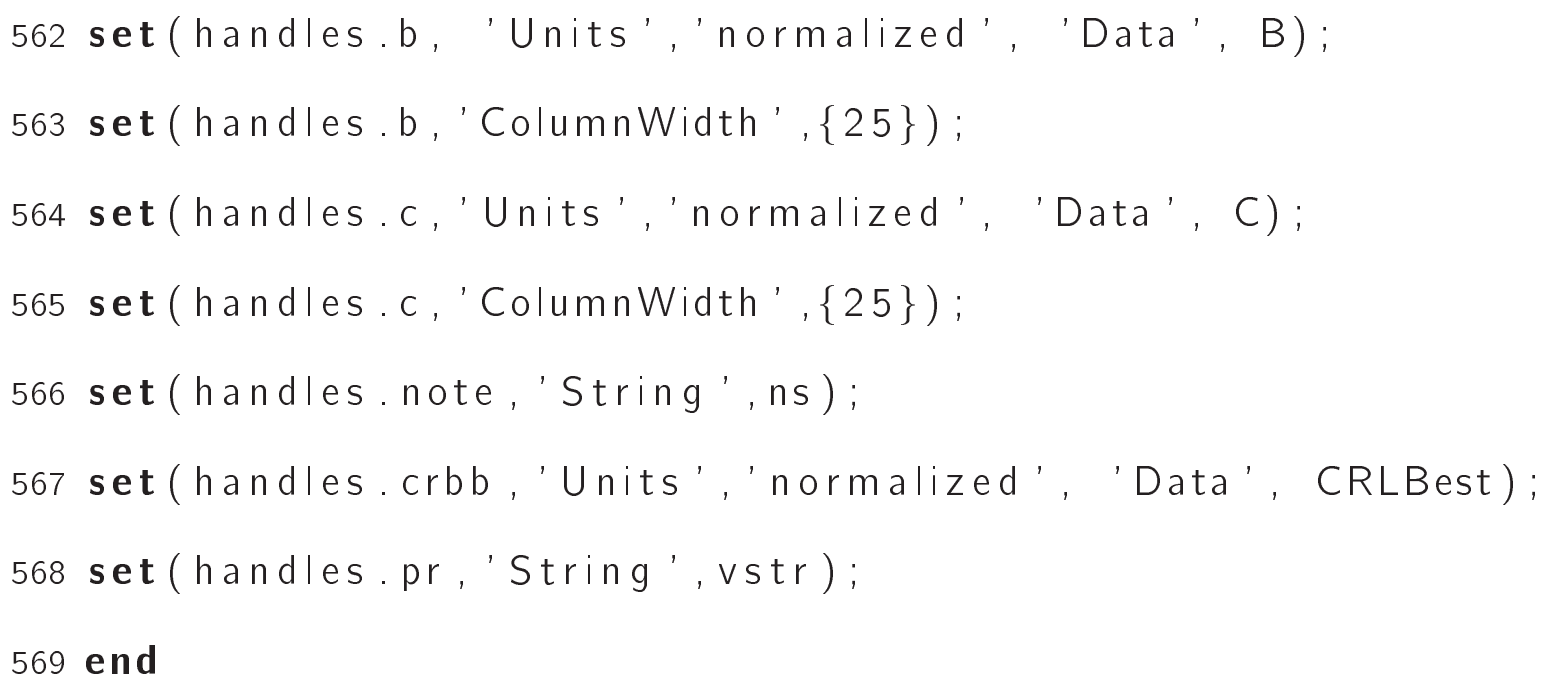




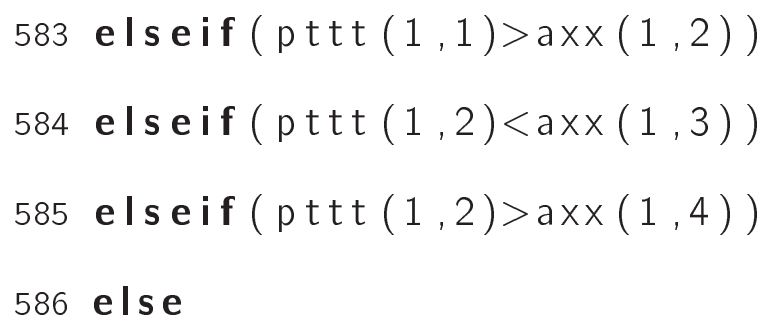

$587 v 6=\operatorname{get}($ handles.draw, 'value ') ;

$588 \vee 7=\operatorname{get}($ handles. vertex, 'value' );

$589 \vee 8=\operatorname{get}($ handles. undirected, 'value' ) ;

590 if $\vee 7==2 \& \& \sim$ isempty (handles. $x y)$

$591 \mathrm{pt}=$ getPoint;

$592 \mathrm{ax}=\mathbf{a x i s}$;

593 if $p t(1)>=a \times(1) \& \& \operatorname{pt}(1)<=a x(2) \& \&$ pt $(2)>=a \times(3) \& \& p t(2)<=a x$

594 handles. edid $=$ get NearestVertex ( pt, handles. $x y)$;

595 guidata (hObject, handles);

596 if handles. eid==handles. edid

597 msgbox ('the starting and ending nodes should be different to draw or delete the edge ', 'Error in draw/delete edge',' error') ;

598 set ( handles. undo, 'enable ', 'off' );

599 end

600 if $\vee 6==1$

601 handles. A ( handles. eid, handles. edid $)=1$;

602 guidata (hObject, handles); 
603 elseif $\vee 6==2$

604 handles. A ( handles. eid, handles. edid $)=0$;

605 guidata (hObject, handles);

606 else

607 end

608 if $\quad \vee 8==1$

609 if handles. edid $\sim$ handles. eid

610 handles. A (handles. edid, handles. eid) = handles. A (handles. eid, handles. edid ) ;

611 guidata (hObject, handles);

612 else

613 handles. A ( handles. edid, handles. eid) $=0$;

614 guidata (hObject, handles);

615 end

616 elseif $\vee 8==2$

617 if handles. edid $==$ handles. eid

618 handles. A (handles. edid, handles. eid) $=0$;

619 guidata (hObject, handles);

620 else

621 end

622 end

623 end

624 cla ; 
625 hold on

626 if isempty (handles. $x y)$

627 plot (handles.axes, handles. xy $(:, 1)$, handles. xy $(:, 2)$, ' ' ')

$628[u x, u y, d x u, d y u, d x I, d y l]=$ plotGraph (handles.A, handles. $x y, a x)$;

$629 \mathrm{n} 2=\operatorname{size}($ handles. $\mathrm{A}, 1)$;

630 plot (handles. axes, ux, uy, 'k-' ,' LineWidth ' ,2)

631 plot (handles. axes, dxu, dyu, 'r: ')

632 plot ( handles. axes, $d x l, d y l$, 'b-' )

$633 \%$ plot (handles.axes, ix, iy, 'ko')

634 plot (handles. axes, handles. xy $(:, 1)$, handles. xy $(:, 2)$, 'k.' $)$

635 for $k=1: \mathrm{n} 2$

636 text (handles. xy $(k, 1)$, handles. xy $(k, 2)$, [', ' num2str $(k)]$, ' Color' , 'k', 'FontSize ',12, 'FontWeight' , 'b ')

637 end

638 end

639 set (gcf, 'WIndowButtonMotionFcn', ', ') ;

640 set (gcf, 'WIndowButtonMotionFcn', \{

(ofigure1_WindowButtonMotionFcn, handles \}) ;

641 elseif $\vee 6==3$

642 set (gcf, 'WIndowButtonMotionFcn', ', ') ;

643 end

644 end

$645 \%--$ Executes on button press in undo. 
646 function undo_Callback (hObject, eventdata, handles)

$647 \vee 9=\operatorname{get}($ handles.draw, 'value');

$648 \vee 10=\operatorname{get}($ handles. vertex, 'value');

$649 \vee 11=\operatorname{get}($ handles. undirected, 'value' ) ;

650 ax=axis ;

651 handles. $x y=$ handles xytemp;

652 handles. $A=$ handles. Atemp;

653 guidata (hObject, handles);

654 if $\vee 9==3$

655 set (gcf, ' WIndowButtonMotionFcn', ' ') ;

656 hold on

657 cla ;

658 if isempty (handles. $x y$ )

659 plot (handles.axes, handles. xy $(:, 1)$, handles. xy $(:, 2)$, ' ' ')

$660[u x, u y, d x u, d y u, d x I, d y l]=\operatorname{plotGraph}($ handles. A, handles. $x y, a x)$;

$661 \mathrm{n} 2=\operatorname{size}($ handles. A, 1$)$;

662 plot (handles. axes, ux, uy, 'k-' , 'LineWidth', 2)

663 plot (handles. axes, dxu, dyu, 'r:')

664 plot (handles.axes, $d x l, d y l$, 'b-')

$665 \%$ plot (handles.axes, ix, iy, 'ko')

666 plot (handles.axes, handles. $x y(:, 1)$, handles. $x y(:, 2)$, 'k.')

667 for $k=1: n 2$ 
668 text (handles.xy $(k, 1)$, handles.xy $(k, 2)$, , ' ' num2str(k) ], 'Color' , 'k', 'FontSize', 12, 'FontWeight' , 'b')

669 end

670 end

671 else

672 hold on

673 cla ;

674 if isempty(handles. $x y$ )

675 plot (handles.axes, handles. xy $(:, 1)$, handles. $x y(:, 2), '$.' )

$676[u x, u y, d x u, d y u, d x l, d y l]=$ plotGraph (handles.A, handles.xy, ax);

$677 \mathrm{n} 2=\operatorname{size}($ handles $. A, 1) ;$

678 plot (handles axes, ux, uy, 'k-' , 'LineWidth' ,2)

679 plot (handles.axes, dxu, dyu, 'r:' )

680 plot (handles.axes, $d x l, d y l$, 'b--')

$681 \%$ plot (handles.axes, ix, iy, 'ko')

682 plot (handles.axes, handles. $x y(:, 1)$, handles. $x y(:, 2)$, 'k.')

683 for $k=1: n 2$

684 text (handles.xy $(k, 1)$, handles.xy $(k, 2)$, [', ' num2str(k) ], 'Color' , 'k', 'FontSize ', 12, 'FontWeight ', 'b')

685 end

686 end

687 set (gcf, 'WIndowButtonMotionFcn', ' ') ; 
688 set (gcf, 'WIndowButtonMotionFcn', \{

(ofigure1_WindowButtonMotionFcn, handles \}) ;

689 end

690 set (handles. undo, 'enable ' , 'off' );

691 if isempty (handles. xy)

692 set (handles. enter, ' enable ', 'off' );

693 set (handles. new, ' enable', ' off' ) ;

694 else

695 end

$696 \%--$ Executes on mouse press over figure background, over a disabled or

$697 \%-$ - inactive control, or over an axes background.

698 function figure1_WindowButtonDownFcn (hObject, eventdata ,

handles )

699 axx=axis ;

700 pttt=getPoint ;

701 if $(\operatorname{pttt}(1,1)<\operatorname{axx}(1,1))$

702 elseif $(\operatorname{pttt}(1,1)>\operatorname{axx}(1,2))$

703 elseif $(\operatorname{pttt}(1,2)<\operatorname{axx}(1,3))$

704 elseif $(\operatorname{pttt}(1,2)>\operatorname{axx}(1,4))$

705 else

706 handles. xytemp = handles. $x y$;

707 handles. Atemp $=$ handles. A; 
708 guidata (hObject, handles);

709 set (handles. enter, 'enable', 'on');

$710 \vee=$ get (handles.draw, 'value');

$711 \vee 1=\operatorname{get}($ handles. $v$ ertex, 'value');

$712 \mathrm{v} 2=\operatorname{get}($ handles. undirected, 'value') ;

$713 \mathrm{pt}=$ getPoint;

714 ax $=$ axis ;

715 if $p t(1)>=a x(1) \& \& \operatorname{pt}(1)<=a x(2) \& \& \operatorname{pt}(2)>=a \times(3) \& \& \operatorname{pt}(2)<=a x$ (4)

716 if $\vee 1==2$

717 if isempty(handles. xy)

718 handles. eid $=$ getNearestVertex (pt, handles. $x y)$;

719 guidata (hObject, handles);

720 end

721 elseif $V==1$

$722 \mathrm{n}=\operatorname{size}($ handles $. x y, 1)$;

723 handles. $x y(n+1,:)=p t$;

724 handles. $A(n+1, n+1)=0$;

725 guidata (hObject, handles);

726 hold on

727 cla ;

728 if isempty (handles. $x y$ )

729 plot (handles.axes, handles.xy $(:, 1)$, handles.xy $(:, 2)$, ' ' ') 
$730[u x, u y, d x u, d y u, d x I, d y l]=$ plotGraph (handles.A, handles. $x y, a x)$;

731 n2 $=$ size (handles. A, 1);

732 plot (handles. axes, ux, uy, 'k-' ,' 'LineWidth ' ,2)

733 plot (handles. axes, dxu, dyu, ' r: ')

734 plot (handles. axes, $d x \mid, d y l$, 'b--' )

$735 \%$ plot (handles.axes, ix, iy, 'ko')

736 plot (handles. axes, handles. xy $(:, 1)$, handles. xy $(:, 2)$, 'k.' $)$

737 for $k=1: n 2$

738 text (handles. xy $(k, 1)$, handles. xy $(k, 2)$, [', ' num2str $(k)]$, ' Color' , 'k', 'FontSize', 12 , 'FontWeight' , 'b')

739 end

740 end

741 set (gcf, 'WIndowButtonMotionFcn', ' ' ) ;

742 set (gcf, 'WIndowButtonMotionFcn', \{

(Qfigure1_WindowButtonMotionFcn, handles \}) ;

743 elseif $\quad v==3$

744 if isempty (handles. xy)

745 handles. id $1=$ get NearestVertex ( pt, handles. $x y)$;

746 guidata (hObject, handles);

747 end

748 set (gcf, 'WIndowButtonMotionFcn', \{

(Qfigure1_WindowButtonMotionFcn, handles \}) ;

749 else 
750 if isempty (handles. xy)

751 id $=$ get NearestVertex (pt, handles. $x y)$;

752 handles. $x y($ id , : $)=[]$;

753 handles. $A($ id,$:)=[]$;

754 handles. $A(:$, id $)=[]$;

755 guidata (hObject, handles);

756 hold on

757 cla ;

758 if isempty (handles. xy)

759 plot (handles. axes, handles. xy $(:, 1)$, handles. xy $(:, 2)$, . ' $)$

$760[u x, u y, d x u, d y u, d x l, d y l]=$ plotGraph (handles.A, handles.xy, ax);

761 n2 $=\operatorname{size}($ handles. A, 1);

762 plot (handles. axes, ux, uy, 'k-' ,' LineWidth', 2) ;

763 plot ( handles. axes, dxu, dyu, 'r: ') ;

764 plot (handles. axes, $d x \mid, d y l$, 'b--' ) ;

765 plot (handles. axes, handles. xy $(:, 1)$, handles. xy $(:, 2)$, 'k. ' )

766 for $k=1: n 2$

767 text ( handles. xy $(k, 1)$, handles. xy $(k, 2)$, [', ' num2str $(k)]$, 'Color' , 'k', 'FontSize', 12, 'FontWeight', 'b' )

768 end

769 end

770 set (gcf, 'WIndowButtonMotionFcn', ', ') ; 
771 set (gcf, 'WIndowButtonMotionFcn', \{

(ofigure1_WindowButtonMotionFcn, handles \}) ;

772 end

773 end

774 end

775 if isempty ( handles. xy)

776 set (handles. new, ' enable', 'on') ;

777 else

778 end

779 set (handles. undo, 'enable', 'on ') ;

780 set (gcf, 'WindowButtonUpFcn', \{ @figure 1 _WindowButtonUpFcn ,

handles \});

781 end

$782 \%--$ Executes on button press in new.

783 function new_Callback (hObject, eventdata, handles)

784 choice $=$ questdlg ('Are you sure you want to clear everything and redraw? This operation cannot be undone.', ...

785 'Draw a New Graph', ...

786 'Yes', 'No', 'No' );

$787 \%$ Handle response

788 switch choice

789 case 'Yes'

790 set ( handles. undo, 'enable' ,' off' ); 
791 cla ;

$792 \times y=[]$

$793 \mathrm{~A}=[]$;

794 eid $=[]$;

795 id $1=[]$;

796 xytemp $=[]$;

797 Atemp $=[]$;

798 enchk $=[]$;

799 enchk $=0$;

800 handles. $x y=x y$;

801 handles. $A=A$;

802 handles . eid=eid;

803 handles. id $1=i d 1$;

804 handles. xytemp $=$ xytemp;

805 handles. Atemp $=$ Atemp;

806 handles. enchk=enchk;

807 handles. prev_scale $=1$;

808 handles.fir_cnt $=[]$;

809 guidata (hObject, handles);

$810 \mathrm{p}=[] ; \mathrm{F} 1=[] ; \mathrm{CRLBmat} 2=[] ; \mathrm{A} 1=[] ; \mathrm{B}=[] ; \mathrm{C}=[] ; \mathrm{ns}=[] ; \mathrm{CRLBest}=[] ; \mathrm{vstr}$ $=[] ; \mathrm{D}=[] ;$

811 set (handles.rev, 'Units', 'normalized' , 'Data', D) ;

812 set (handles. poles, 'Units', 'normalized', 'Data', p); 


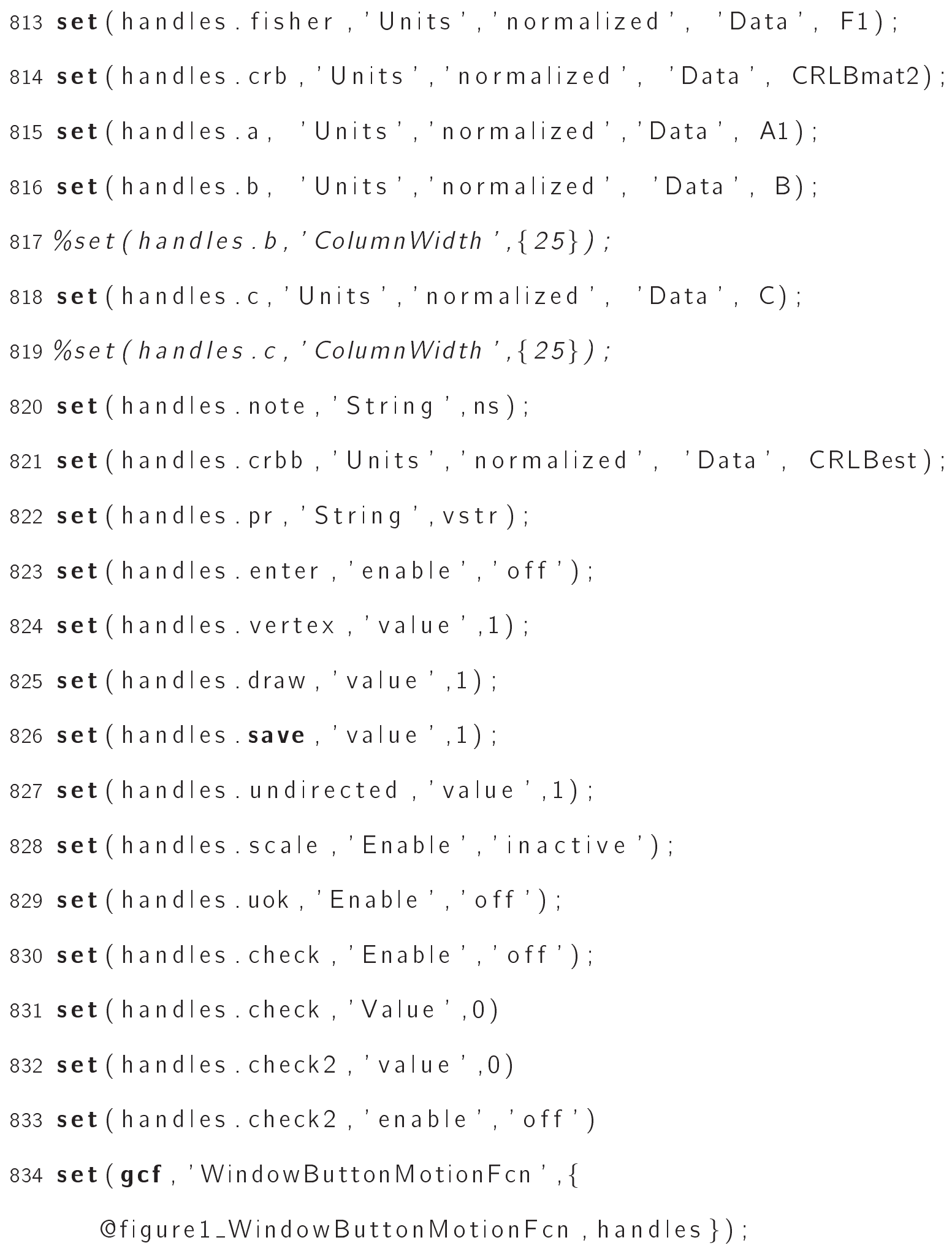




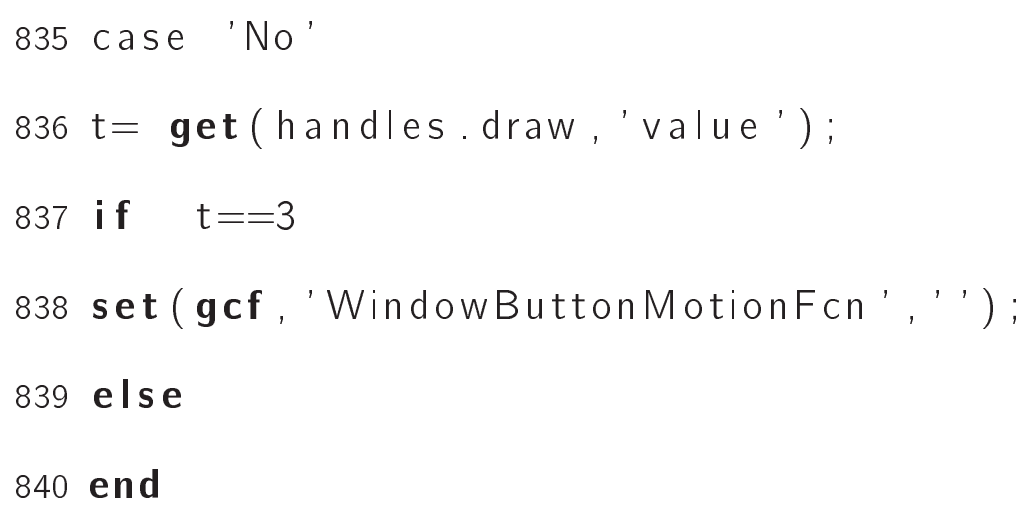


$857[\mathrm{~m}, \mathrm{o}]=\mathbf{s i z e}(\mathrm{h}$ andles. xy $)$;

858 if in_act $>m$ \%if the input is higher than the number of nodes $859 \%$ this is the first line of the msgbox

$860 \operatorname{msgbox} T$ ext $\{1\}=$ 'You have entered a value that is higher than the number of nodes at actuation location input';

$861 \%$ this command creates the actual message box 862 msgbox (msgboxText, 'Input number incorrect', ' error');

863 elseif isempty (in_act) \%if the input is not a number $864 \%$ this is the first line of the msgbox 865 msgboxText $\{1\}=$ 'You have tried to input something that is NOT a number at actuation location input.' ; $866 \%$ this command creates the actual message box 867 msgbox (msgboxText, 'Input not a number', 'error' ) ; 868 elseif isnan ( in_act) \%if the input is not a number $869 \%$ this is the first line of the msgbox $870 \operatorname{msgbox} T \operatorname{ext}\{1\}=$ 'Nothing is entered at actuation location input.' ;

$871 \%$ this command creates the actual message box 872 msgbox(msgboxText, 'Input empty', ' error' ); 873 elseif in_meas $>m \%$ if the input is higher than the number of nodes

$874 \%$ this is the first line of the msgbox 
875 msgboxText $\{1\}=$ 'You have entered a value that is higher than the number of nodes at measurement location input' ;

$876 \%$ this command creates the actual message box

877 msgbox(msgboxText, 'Input number incorrect', 'error');

878 elseif isempty (in_meas) \%if the input is not a number

$879 \%$ this is the first line of the msgbox

$880 \operatorname{msgbox} T$ ext $\{1\}=$ 'You have tried to input something that is

NOT a number at measurement location input.' ;

$881 \%$ this command creates the actual message box

882 msgbox(msgboxText, 'Input not a number', 'error' );

883 elseif isnan (in_meas) \%if the input is not a number

$884 \%$ this is the first line of the msgbox

$885 \operatorname{msgbox} T$ ext $\{1\}=$ 'Nothing is entered at measurement location

input input. ' ;

$886 \%$ this command creates the actual message box

887 msgbox(msgboxText, 'Input empty', ' error' );

888 elseif isempty ( in_noise) \%if the input is not a number

$889 \%$ this is the first line of the msgbox

$890 \operatorname{msgbox} T$ ext $\{1\}=$ 'You have tried to input something that is

NOT a number at Noise varience input.' ;

$891 \%$ this command creates the actual message box

892 msgbox (msgboxText, 'Input not a number', 'error' ) ;

893 elseif isnan (in_noise) \%if the input is not a number 
$894 \%$ this is the first line of the msgbox

895 msgboxText $\{1\}=$ 'Nothing is entered at Noise input.';

$896 \%$ this command creates the actual message box

897 msgbox(msgboxText, 'Input empty', 'error');

898 else

$899 \%$ Call the matrix function to calculate several outputs for the

$900 \%$ current input.

901 handles. fix $1=\operatorname{str} 2$ double ( get (handles. scale, 'String') );

902 guidata(hObject, handles);

$903 \mathrm{sc}=$ uokk;

904 en $=1$;

$905 \mathrm{c} 2=\boldsymbol{g e t}($ handles. check2, 'value');

906 if $\mathrm{c} 2==1$

907 fixval $=$ handles. fix 1 ;

908 fix $=1$;

909 else

$910 \mathbf{f i x}=0$;

911 fixval $=1$;

912 end

913 [mat2 , p, r, F1, CRLBmat2, A1, B, C, vstr , xymid, ns, CRLBest, posi, kk, D, errchk] = matrix (handles. xy, handles. A, in_act, in_meas, in_noise, sc, en, fix, fixval, handles.prev_scale); 
914 handles. prev_scale $=k k$;

915 guidata (hObject, handles);

916 if $\operatorname{errchk}==1$

917 errordlg('Scaling factor given by user in not valid', 'error');

918 else

$919[\mathrm{~m} 3, \mathrm{o} 3]=\mathbf{s i z e}($ handles. A $)$;

920 count $=0$;

921 cla ;

922 hold on

$923 \% \%$ Display the edge weights on respective edges.

924 for $e=1: m 3$

925 for $f=e+1: m 3$

926 if handles. $A(e, f)==1$

927 count $=$ count +1 ;

928 text (xymid (count, 1 ), xymid (count, 2 ) , [' ' num 2str(A1(e,f)) ] ,' Color' , 'k', 'FontSize', 8, 'FontWeight' , 'b' );

929 else

930 count $=$ count +1 ;

931 end

932 end

933 end

$934 \% \%$ Display and plot all outputs on GUI.

935 if isempty (handles.xy) 
936 plot (handles.axes, handles.xy $(:, 1)$, handles. xy $(:, 2), '$.' $)$

937 [ ux, uy, dxu, dyu, dxl, dyl] = plotGraph (handles.A, handles. $x y$ );

$938 \mathrm{n} 2=\operatorname{size}($ handles. A, 1$)$;

939 plot (handles. axes, ux, uy, 'k-' , 'LineWidth' ',2)

940 plot (handles. axes, dxu, dyu, ' $r$ :' )

941 plot (handles.axes, $d x l, d y l, ' b-')$

$942 \%$ plot (handles.axes, ix, iy, 'ko')

943 plot (handles.axes, handles. xy $(:, 1)$, handles. xy $(:, 2)$, 'k.')

944 for $k=1: n 2$

945 text (handles.xy $(k, 1)$, handles.xy $(k, 2)$, [', ' num2str(k)], 'Color' , 'k', 'FontSize',12, 'FontWeight' , 'b ')

946 end

947 end

948 text (handles.xy (in_act, 1$)$, handles. xy (in_act, 2), [' ' num 2str( in_act)] , 'Color', 'b ', 'FontSize',12, 'FontWeight' , 'b ')

949 text (handles.xy (in_meas, 1), handles.xy (in_meas, 2), [' ' num2str (in_meas) ], 'Color', 'r' , 'FontSize',12, 'FontWeight' , 'b')

950 text (handles.xy (posi, 1), handles.xy (posi, 2), [', ', num2str(posi)

], 'Color' , 'g', ' FontSize', 12, 'FontWeight ', 'b' )

951 text (7,9.15, 'Blue- Actuation', 'Color' ,'b' , 'FontSize' ,8)

952 text ( $7,8.83$, 'Red- Measurement ', 'Color', 'r' , 'FontSize' ,8)

953 text $(7,8.51$, 'Green- Best Measurement ', 'Color' , 'g' , 'FontSize ' 
if Iength $(d \times \mid)>=2$

955 if $\quad$ isnan $(d \times I(1,1)+d x I(2,1))$

956 text $(0.52,9.47$,' Directed edges:' ,' 'FontSize', 8)

957 text $(0.52,9.15$, '-- Blue large to small node', 'Color' , 'b', '

FontSize', 8 )

958 text $(0.52,8.83$, , - Red small to large node', 'Color', 'r', '

FontSize', 8 )

959 else

960 end

961 if length $(\mathrm{d} \times \mathrm{u})>=2$

962 if isnan $(\mathrm{d} \times \mathrm{u}(1,1)+\mathrm{d} \times \mathrm{u}(2,1))$

963 text (0.52,9.47,' Directed edges:' ,' FontSize', 8)

964 text $(0.52,9.15$, '-- Blue large to small node', 'Color' , 'b', '

FontSize', 8 )

965 text $(0.52,8.83$, , - Red small to large node', 'Color', 'r', '

FontSize', 8 )

966 else

967 end

968 else

969 end

970 set (handles. scale, 'Units', ' normalized', 'String', kk) ;

971 set (handles.rev, 'Units', 'normalized' , 'Data', D) ;

972 set (handles. poles, 'Units', ' normalized' , 'Data', p) ; 


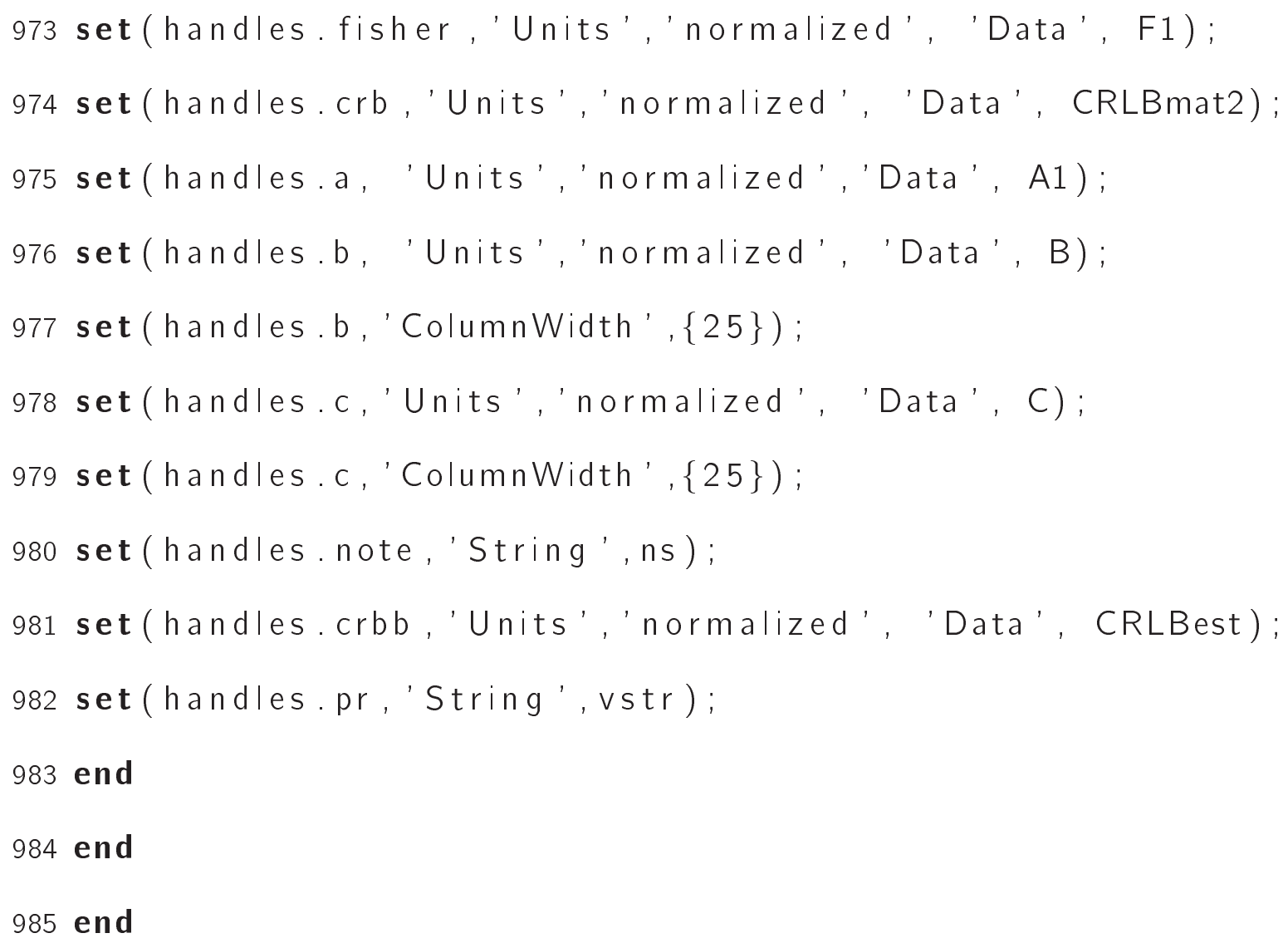




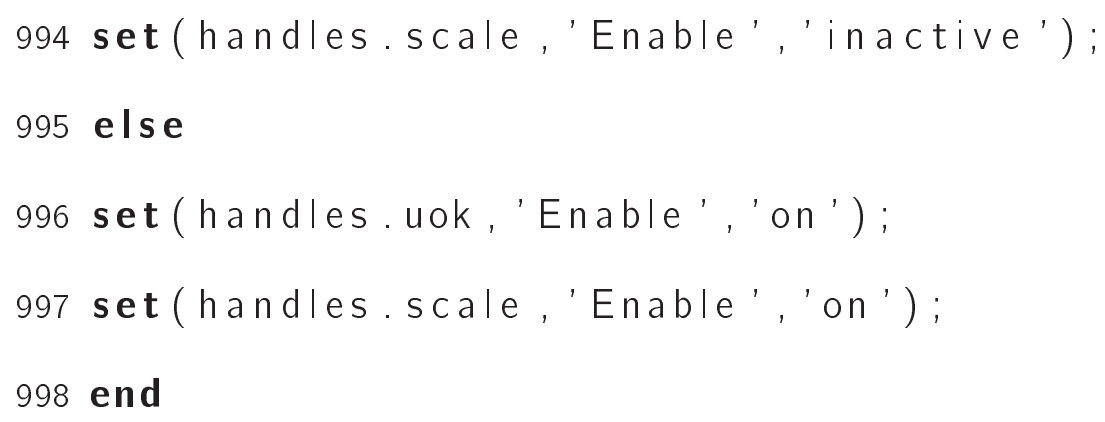


$1017 \%$ set (handles.uok, 'Enable', 'off');

1018 set (handles. scale, 'Enable', ' inactive') ;

1019 set (handles .uok, 'Enable', ' off'); 


\section{APPENDIX B}

\section{MATRIX FUNCTION: RETURNS DIFFERENT OUTPUTS IN GUI}


$1 \% \%$ This function calculates different outputs for the current user input

$2 \% \%$ and retuns to display $\% \%$

3 function [mat2, p, r, F1, CRLBmat2, A1, B, C, vstr, xymid, ns, CRLBest, posi, $k, D$, errchk] = matrix (xy, A, in_act, in_meas, in_noise, sc, en, fix, fixval, prev_scale )

$4 \% \%$ Get the $x y$ locations and calcuate the distance between the vertices

$5[\mathrm{~m}, \mathrm{o}]=\operatorname{size}(x y) ;$

$6 \mathrm{niCr}=\mathrm{m}^{\wedge} 2$;

7 xymid $=[]$;

$8 \mathrm{t} 1=1$;

9 for $e=1: m$

10 for $f=e+1: m$

11 xymid $(\mathrm{t} 1,:)=(x y(e,:)+x y(f,:)) / 2$;

$12 \mathrm{t} 1=\mathrm{t} 1+1$;

13 if $\mathrm{t} 1==\mathrm{niCr}+1$;

14 end

15 end

16 end

$17[\mathrm{~m} 4,04]=\mathbf{s i z e}($ xymid $)$;

$18 \mathrm{t}=1$;

19 temp $=[n i C r, 2]$; 
20 tempsq $=[\mathrm{niCr}, 2]$;

21 for $e=1: m$

22 for $f=1: m$

$23 \operatorname{temp}(\mathrm{t},:)=x y(\mathrm{e},:)-\mathrm{xy}(\mathrm{f},:) ;$

$24 \mathrm{t}=\mathrm{t}+1$;

25 if $\mathrm{t}==\mathrm{niCr}+1$;

26 end

27 end

28 end

29 tempsq $=$ temp.^ 2 ;

30 tempsqrt $=(\operatorname{tempsq}(:, 1)+\operatorname{tempsq}(:, 2)) \cdot{ }^{\wedge}(0.5) ;$

31 matemp $=\operatorname{vec} 2$ mat (tempsqrt, $m)$

32 mat $=[]$;

33 one $=$ eye $(\mathrm{m})$;

34 mat $1=$ one + matemp ;

35 for $j=1: m$

36 for $k=1: m$

$37 \operatorname{mat}(j, k)=(\operatorname{mat} 1(j, k))^{\wedge}-1$;

38 end

39 end

40 mat2 $=$ mat.$* A$;

$41 \% \%$ Form the Laplacian matrix L \% \% 
$42 \mathrm{Ln}=(-1) *$ mat2; $\%$ Ln, the values of the input matrix to negative values

$43 \mathrm{I}=$ eye (size $(\operatorname{Ln})) ; \%$ l, identity matrix, has size of the input matrix

$44 \mathrm{dg}=\operatorname{sum}\left((\operatorname{Ln})^{\prime}\right) ; \% d g$, vector that stores the values of the row sums of the input matrix $\mathrm{Ln}$

$45 \mathrm{dg} 1=-\mathrm{dg}$;

$46 \% k=$ numel $($ num2str $($ int32(var) $))$

$47 \mathrm{DD}=\mathbf{d i a g}(\mathrm{dg}, 0) ; \% \mathrm{D}$, diagonal matrix of zeros and a main diagonal dg (already defined above)

$48 \mathrm{~L} 1=\mathrm{Ln}+((-1) * \mathrm{DD}) ; \% \mathrm{~L}$, Iaplacian matrix with the inverted input entries and diagonal entries equal to the negative of row sums.

49 if $\quad$ en $==1$

$50 \mathrm{~L} 3=\mathrm{L} 1 * \mathrm{SC}$;

$51 \mathrm{k}=\mathrm{sC}$;

52 else

53 end

54 if $\mathbf{f i x}==1$

$55 L 3=L 1 *$ fixval;

$56 k=$ fixval;

57 elseif $\mathbf{f i x}==0$

58 var $=\max (\operatorname{dg} 1(:))$; 


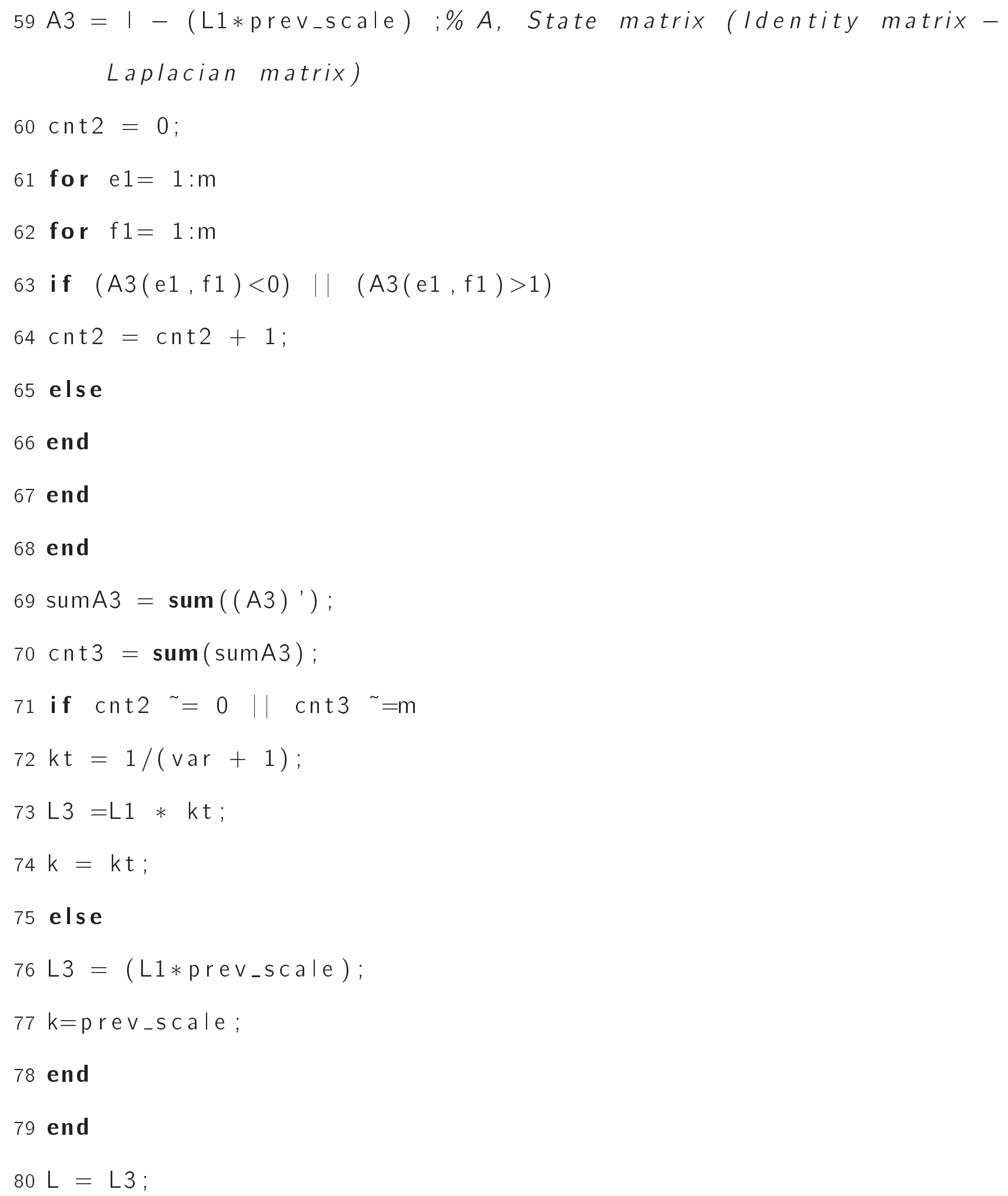
$80 \mathrm{~L}=\mathrm{L} 3$; 
$81 \mathrm{~A} 2=\mathrm{I}-\mathrm{L} ; \% \mathrm{~A}$, State matrix (Identity matrix - Laplacian matrix)

$82 \mathrm{cnt}=0$;

83 for $e=1: m$

84 for $f=1: m$

85 if $(A 2(e, f)<0) \quad \| \quad(A 2(e, f)>1)$

$86 \mathrm{cnt}=\mathrm{cnt}+1$;

87 else

88 end

89 end

90 end

$91 \operatorname{sum} A 2=\operatorname{sum}\left((A 2)^{\prime}\right) ;$

92 cnt $1=\operatorname{sum}(\operatorname{sum} A 2)$;

93 if cnt $\sim=0||$ cnt $1 \stackrel{\sim}{=}=\mathrm{m}$

94 errchk $=1$;

95 else

96 errchk $=0$;

97 end

$98 \mathrm{~A} 1=\mathrm{I}-\mathrm{L}$;

$99[\mathrm{D}, \mathrm{V}]=\operatorname{eigs}(\mathbf{f} \mathbf{u} \mathbf{I I}(\mathrm{A} 1))$;

$100 \mathrm{~B}=\operatorname{zeros}(\mathrm{m}, 1) ; \%$ ei, vector of zeros, has a row size of the input matrix Lin

$101 \mathrm{~B}($ in_act $)=1 ; \%$ Changing the first entry of ei vector to 1 


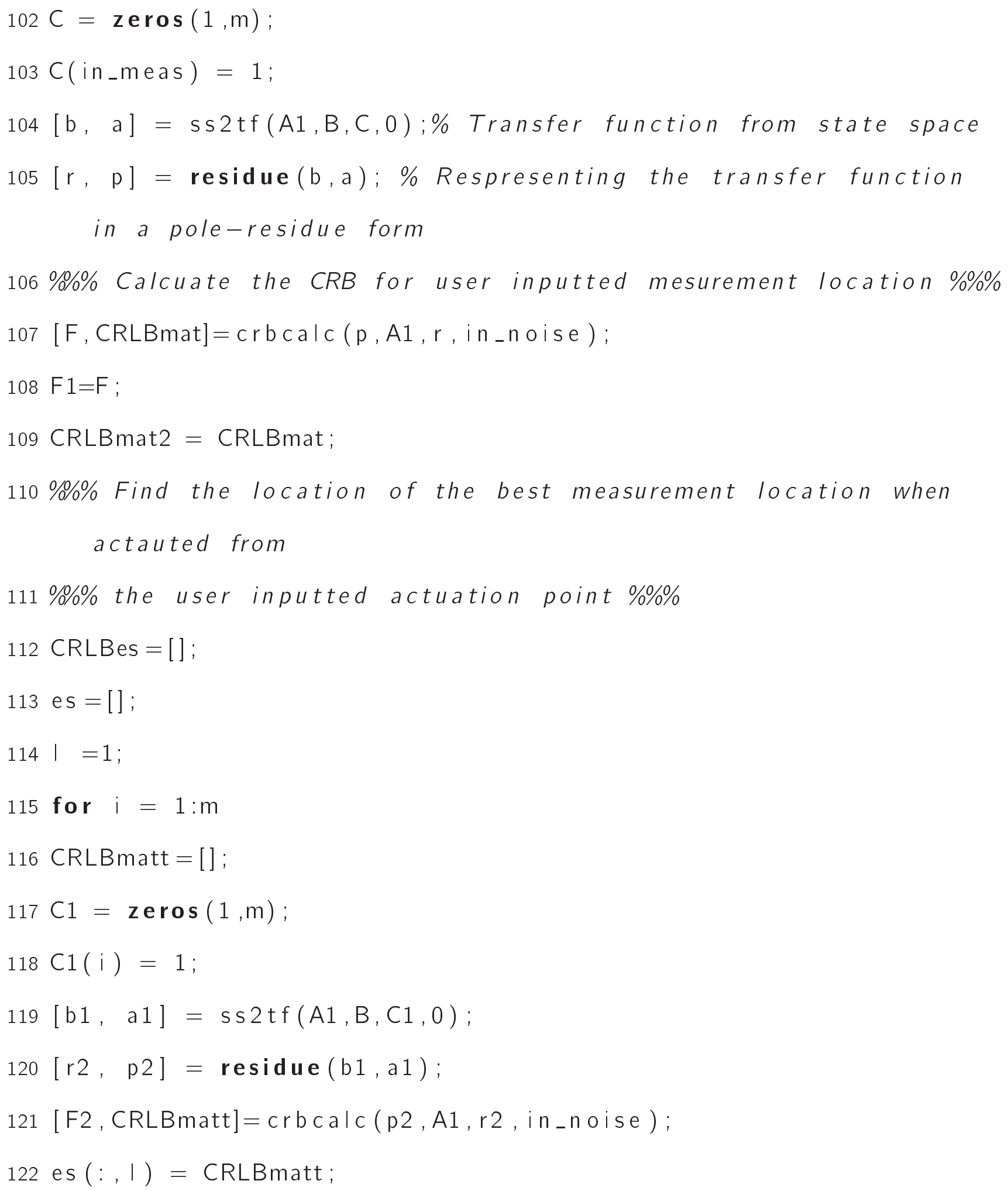


$123|=|+1$;

\section{4 end}

125 CRLBes $=$ es $(2,:)$;

$126 \operatorname{CRLBes}(1$, in_act $)=1000$;

127 least $=[]$;

128 posi $=[]$;

129 least $=\min (\operatorname{CRLBes}(:))$;

130 for $\mathrm{i}=1: \mathrm{m}$

131 if $\operatorname{CRLBes}(1, \mathrm{i})==$ least

132 posi $=i$;

133 else

134 end

135 end

136 CRLBest $=[]$;

137 Best $=[]$;

$138 \mathrm{C} 2=\operatorname{zeros}(1, \mathrm{~m})$;

$139 \mathrm{C} 2($ posi $)=1$;

$140[\mathrm{~b} 2, \mathrm{a} 2]=\operatorname{ss} 2 \mathrm{tf}(\mathrm{A} 1, \mathrm{~B}, \mathrm{C} 2,0)$;

$141[\mathrm{r3}, \mathrm{p} 3]=$ residue $(\mathrm{b} 2, \mathrm{a} 2)$;

$142[F 3$, Best $]=\operatorname{crbcalc}(p 3, A 1, r 3$, in_noise $)$;

143 CRLBest $=$ Best ;

$144 \% \%$ Calculate the Pole - Residue form \%\%

$145 \mathrm{p} 1=\mathrm{p}$; 
$146 r 1=r$;

147 for $\mathrm{i}=1: \mathrm{m}$

148 if $p(i, 1)<0$ \&\& $r(i, 1)<0$

$149 \mathrm{p} 1(\mathrm{i}, 1)=-\mathrm{p} 1(\mathrm{i}, 1)$;

$150 \mathrm{r} 1(\mathrm{i}, 1)=-\mathrm{r} 1(\mathrm{i}, 1)$;

$151 \operatorname{vstr}\{i\}=$ sprintf $\left({ }^{\circ} \% f \backslash n \% s \backslash n \% s \% f \% c \backslash n{ }^{\prime}, r 1(i, 1)\right.$, '', ' $\left.(z+,, p 1(i, 1), ')^{\prime}\right)$;

152

153 elseif $p(i, 1)<0 \& \& r(i, 1)>0$

$154 \mathrm{p} 1(\mathrm{i}, 1)=-\mathrm{p} 1(\mathrm{i}, 1)$;

$155 \operatorname{vstr}\{i\}=$ sprintf $\left({ }^{\prime} \% \mathrm{f} \backslash \mathrm{n} \% \mathrm{~s} \backslash \mathrm{n} \% \mathrm{~s} \% \mathrm{f} \% \mathrm{c} \backslash \mathrm{n}^{\prime}, \mathrm{r}(\mathrm{i}, 1),^{\prime}+\right.$ ', ' $\left.(z+,, p 1(i, 1), ')^{\prime}\right)$;

156

157 elseif $p(i, 1)>0 \& \& r(i, 1)>0$

$158 \operatorname{vstr}\{i\}=$ sprintf $\left({ }^{\prime} \% f \backslash n \% s \backslash n \% s \% f \% c \backslash n^{\prime}, r(i, 1),{ }^{\prime}+\right.$ ', ' $(z-,, p(i, 1)$, ' $)$ ') ;

159 else

$160 \operatorname{rr}(\mathrm{i}, 1)=-\mathrm{r} 1(\mathrm{i}, 1)$;

$161 \operatorname{vstr}\{i\}=$ sprintf $\left({ }^{\prime} \% f \backslash n \% s \backslash n \% s \% f \% c \backslash n{ }^{\prime}, r 1(i, 1),{ }^{\prime}-\right.$ ', ' $\left.(z-,, p(i, 1),)^{\prime}\right)$;

162 end

163 end 
$164 \mathrm{~ns}=$ sprintf $\left({ }^{\circ} \% \mathrm{~s} \% \mathrm{u} \backslash \mathrm{n} \% \mathrm{~s} \% \mathrm{u} \backslash \mathrm{n} \% \mathrm{~s}\right.$ ', ' The dynamics when actuated at node:', in_act,' are best observed at node: ', posi, 'The Cramer Rao bounds at this node are given below');

$165 \%$ the function calculates the Fisher matrix and CRB \%\%

166 function $[F, C R L B m a t]=\operatorname{crbcalc}(p, A 1, r$, in_noise $)$

167 CRLBmat $=[]$;

168 temp $1=[]$;

$169[\mathrm{n} 2, \mathrm{~m} 2]=\operatorname{size}(\mathrm{A} 1)$;

170 for $i=1: n 2$

171 for $j=1: n 2$

$172 X A(i, j)=1 /(1-(p(i) * p(j)))$;

173 end

174 end

175 for $i=1: n 2$

176 for $j=1: n 2$

$177 X B(i, j)=(r(i) * p(j)) /\left((1-(p(i) * p(j)))^{\wedge} 2\right)$;

178 end

179 end

$180 X C=X B^{\prime}$;

181 for $i=1: n 2$

182 for $j=1: n 2$

$183 X D(i, j)=\left(r(i) * r(j) *\left(1-(p(i) * p(j))^{\wedge} 2\right)\right) /\left((1-(p(i) * p(j)))^{\wedge} 4\right)$;

184 end 
185 end

$186 \mathrm{FA}=$ vertcat $(\mathrm{XA}, \mathrm{XB})$;

$187 \mathrm{FB}=\operatorname{vertcat}(\mathrm{XC}, \mathrm{XD})$;

$188 \mathrm{~F}=[\mathrm{FA} F \mathrm{FB}]$

189 for $i 1=1:$ length $(A 1)$

$190 \operatorname{temp} 1(\mathrm{i} 1)=\left(\left(1-\left(\mathrm{p}(\mathrm{i} 1)^{\wedge} 2\right)\right)^{\wedge} 3\right) /\left(\left(\mathrm{r}(\mathrm{i} 1)^{\wedge} 2\right) *\left(1+\left(\mathrm{p}(\mathrm{i} 1)^{\wedge} 2\right)\right)\right)$;

191 end

192 CRLBmat $=\left(\text { temp1 } * \text { in } \_ \text {noise }\right)^{\prime} ;$

193 end

194 end 


\section{APPENDIX C}

OTHER FUNCTIONS REQUIRED FOR PLOTTING NETWORKS 


\section{C.1. PLOT GRAPH FUNCTION}

$1 \% \%$ Function will plot the graph structure on the GUI axis \%\%

2

3 function $[u x, u y, d x u, d y u, d x l, d y l]=\operatorname{plotGraph}(A, x y, a x)$

4

5

7

8

9

$$
\begin{aligned}
& \text { if nargin }<3 \\
& \text { ax }=\text { axis; } \\
& \text { else } \\
& \min x=\min ([\operatorname{ax}(1) ; x y(:, 1)]) ; \\
& \operatorname{maxx}=\max ([\operatorname{ax}(2) ; x y(:, 1)]) ; \\
& \operatorname{miny}=\min ([\operatorname{ax}(3) ; x y(:, 2)]) ; \\
& \operatorname{maxy}=\max ([\operatorname{ax}(4) ; x y(:, 2)]) ; \\
& \operatorname{ax}=[\min x \operatorname{maxx} \operatorname{miny} \operatorname{maxy}] ;
\end{aligned}
$$

end

$[u x, u y, d x u, d y u, d x I, d y l]=\operatorname{dgplot}(A, x y)$;

$$
\text { axis }(a x) \text {; }
$$

function $[u x, u y, d x u, d y u, d x I, d y l]=\operatorname{dgplot}(A, x y)$

$\mathrm{n}=\operatorname{size}(A, 1)$;

$A=\max (0, \min (1, \mathbf{c e i l}(\mathbf{a b s}(A))))$;

$\mathrm{uA}=\mathrm{A} \cdot * \mathrm{~A}^{\prime} \cdot *(1-\mathbf{e y e}(\mathrm{n}))$

$[u x$, uy $]=\operatorname{makeXY}(u A, x y)$;

$\%$ undirected edges

$$
(2-\text { way })
$$

$[\mathrm{d} x u, \mathrm{dyu}]=\operatorname{makeXY}(\mathbf{t} \mathbf{r i u}(\mathrm{A}-\mathrm{uA}), \mathrm{xy}) ; \%$ directed edges

$$
(1-w a y, \quad s m 2 / g)
$$




$$
(1-\text { way, } \lg 2 \mathrm{sm})
$$

$$
\begin{aligned}
& \%[i x, i y]=\operatorname{makeXY}(A . * \text { eye }(n), x y) \quad \% \text { self-connecting } \\
& \text { edges }
\end{aligned}
$$

$$
\text { function }[x, y]=\operatorname{makeXY}(A, x y)
$$

$$
\mathrm{x}=\mathrm{NaN} \text {; }
$$$$
\mathrm{y}=\mathrm{NaN} \text {; }
$$

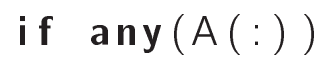

\section{C.2. GET NEAREST VERTEX FUNCTION}

$1 \% \%$ This function returns the nearest vertex ID number which is nearer to 


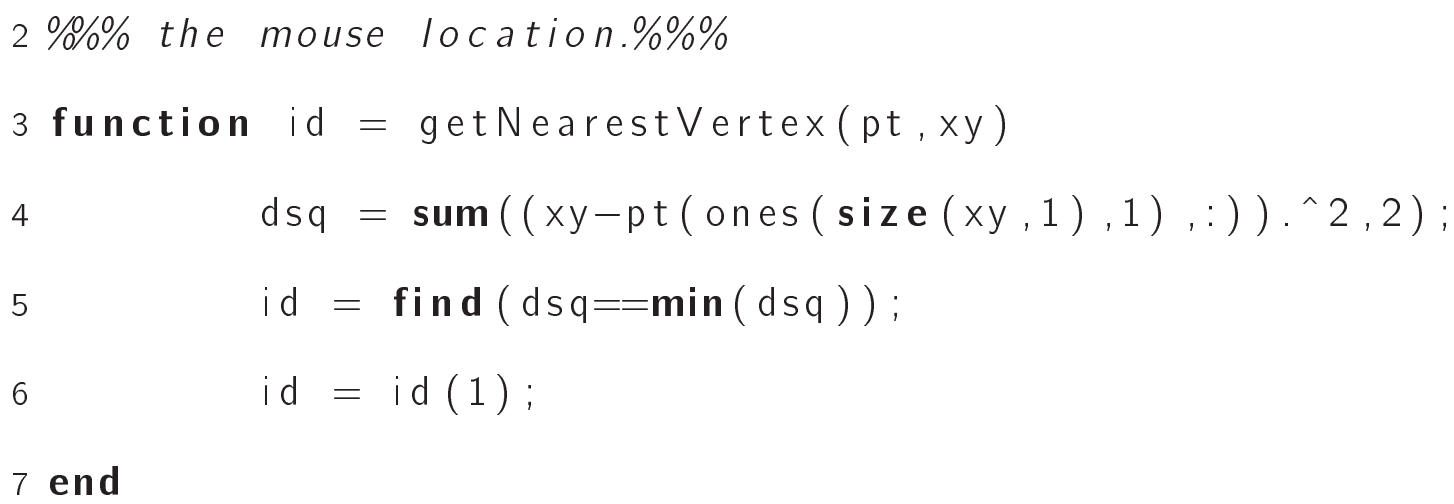

1 function $[x, y]=\operatorname{makeXY} 1(A, x y)$

2

$$
\mathrm{x}=\mathrm{NaN} \text {; }
$$$$
\mathrm{y}=\mathbf{N a N} \text {; }
$$

4

$$
\text { if any }(A(:))
$$

5

$[i i, j j]=$ find $(A)$;

$\mathrm{m}=\operatorname{length}(\mathrm{i} i) ;$

xmat $=[x y(i i, 1) \quad x y(j j, 1)]^{\prime} ;$

ymat $=[x y(i i, 2) \quad x y(j j, 2)]^{\prime} ;$

$\mathrm{x}=[\mathrm{xmat} ; \operatorname{NaN}(1, \mathrm{~m})] ; \quad \mathrm{x}=\mathrm{x}(:)$;

$\mathrm{y}=[\mathrm{ymat} ; \boldsymbol{N a N}(1, \mathrm{~m})] ; \mathrm{y}=\mathrm{y}(:) ;$

11

end

12 end

C.4. GET POINT FUNCTION 
$1 \% \%$ This function returns the location of the mouse pointer on GUI axis. $\% \% \%$

2 function $p t=$ getPoint ()

3

$$
\text { cpt }=\text { get }(\text { gca , 'CurrentPoint'); }
$$

4

$p t=\operatorname{cpt}(1,1: 2)$;

5

latch $=0.01$;

6

if isnan ( latch)

7

$p t=\operatorname{round}(p t / I a t c h) * \mid a t c h$;

8

end

9 end 


\section{BIBLIOGRAPHY}

[1] http://www.ee.unt.edu/public/wan/.

[2] S. Boyd, P. Diaconis, and L. Xiao, Fastest mixing markov chain on a graph, SIAM Review $46,667-689$.

[3] R. Dhal, S. Roy, Y. Wan, A. Saberi, and P. Corrigan, Majorizations for the eigenvectors of graph-adjacency matrices, submitted.

[4] Mary J. Dunlop and Richard M. Murray, Towards biological system identification: fast and accurate estimates of parameters in genetic regulatory networks, Proceedings of Conference on Decision and Control, December 2006.

[5] George Casella E.L. Lehmann, Theory of Point Estimation (2nd edition), Springer-Verlag New York, Inc., 1998.

[6] Byron Ellis and Wing Hung Wong, Learning causal bayesian network structures from experimental data, Journal of the American Statistical Association 103 (2008).

[7] M Fiedler, Absolute algebraic connectivity of trees, Linear and Multilinear Algebra 26, 85-106.

[8] M. Fiedler, Eigenvectors of acyclic matrices, Czechoslovak Mathematics Journal 100, $607-618$.

[9] J.D. George, R.R. Muise, and J.S. Abel, Simultaneous estimation of time-of-arrival, pole, and amplitude parameters for transient exponential signals, Proceedings of Acoustics, Speech, and Signal Processing, 1992. ICASSP-92., 1992 IEEE International Conference on, March 1992.

[10] Robert F Guratzsch and Sankaran Mahadevan, Structural health monitoring sensor place- 
ment optimization under uncertainty, American Institute of Aeronautics and Astronautics(AIAA Journal 2010) 48 (2010), no. 7, 1281-1289.

[11] Cramer Harald, Mathematical Methods of Statistics, Princeton Univ press, Princeton, NJ, 1946.

[12] H Hashimoto, Distributed cyber attack detection for power network systems, Proceedings of 50th IEEE Conference on Decision and Control and European Control Conference (CDC-ECC), December 2011.

[13] Martijn P Van Den Heuvel, Rene C W Mandl, Cornelis J Stam, Rene S Kahn, and Hilleke E Hulshoff Pol, Aberrant frontal and temporal complex network structure in schizophrenia: a graph theoretical analysis, Journal of Neuroscience, November 2010.

[14] Lieven De Lathauwer Jean-Michel Papy and Sabine Van Huffel, Common pole estimation in multi-channel exponential data modeling, 2006.

[15] Veenadhar Katragadda, Marigona Bokshi, Yan Wan, and Xinrong Li, An interactive tool to investigate the inference performance of network dynamics from data, Submitted to the Proceedings of Infotech@Aerospace 2012, American Institute of Aeronautics and Astronautics (AIAA) (California, USA), June 19 - 212012.

[16] Steven M. Kay, Cramer-rao lower bound, PH sinal processing series, no. 5035, Prentice Hall, Inc. Upper Saddle River, New Jersy 07458, 1993.

[17] Haoni Li, Nan Wang, Ping Gong, Edward J Perkin, and Chaoyang Zhang, Learning the structure of gene regulatory networks from time series gene expression data, Proceedings of The 2010 International Conference on Bioinformatics and Computational Biology (BIOCOMP 2010): Genomics (Las Veagas, NV, USA), July 2010.

[18] I.A. Maraziotis, A. Dragomir, and A. Bezerianosi, Gene networks reconstruction and time-series prediction from microarray data using recurrent neural fuzzy networks, IET 
Systems Biology 1 (2007), 41-50.

[19] L.T. McWhorter and L.L. Scharf, Cramer-rao bounds for deterministic modal analysis, IEEE Trans. Signal Proc. SP-41 (1993), 1847-1862.

[20] Jean-Philippe Ovarlez, Cramer rao bound computation for velocity estimation in the broad-band case using the mellin transform, Acoustics, Speech, and Signal Processing, 1993. ICASSP-93., 1993 IEEE International Conference on, April 1993.

[21] Cynthia Phillips and Laura Painton Swiler, A graph-based system for networkvulnerability analysis, Workshop on New security paradigms NSPW 98 (1998), 1998.

[22] Li Shi-Hua and Tang De-Shan, Power grid crises management and empirical research on small disturbance character of power grid, Proceedings of Power and Energy Engineering Conference, 2009. APPEEC 2009, March 2009.

[23] Rakesh Kumar Sinha and Amit Kumar Ray, Effect of acute and chronic heat exposure on frequency of eeg components in different sleep-wake state in young rats, Iranian Biomedical Journal 8 (2004), no. 2, 69-75.

[24] V. Anne Smith, Jing Yu, Tom V. Smulders, Alexander J. Hartemink, and Erich D. Jarvis, Computational inference of neural information flow networks, PLoS Computational Biology 2 (2006), 1436-1449.

[25] Harry L. Van Trees, Detection, Estimation and Modulation Theory, Part I, John Wiley and Sons, 1968.

[26] Y. Wan, K. Numuduri, S. Akula, and M. Varanasi, The impact of multi-group multilayer network structure on the performance of distributed consensus building strategies, International Journal of Robust and Nonlinear Control (available online before print, February 2012).

[27] Y. Wan and S. Roy, On inference of network time constants from impulse response 
data: graph-theoretic cramer-rao bounds, Proceedings of the 48th IEEE Conference on Decision and Control (Shanghai, China), December 2009.

[28] Y. Wan, S. Roy, and A. Saberi, Designing spatially-heterogeneous strategies for the control of virus spread, IET Systems Biology 2 (2008), 184-201.

[29] Yan Wan and Sandip Roy, On inference of network time constants from impulse response data: Graph-thoretic cramer-rao bounds, Proceedings of Joint 48th IEEE Conference on Decision and Control 28th Chinese Control Conference (Shanghai, P.R. China), December 2009.

[30] M Xue, S Roy, Y Wan, and S Das, Security and vulnerability of cyber-physical infrastructure networks: A control-theoretic approach, (2012).

[31] Ting Hua Yi, Hong Nan Li, and Ming Gu, Optimal sensor placement for structural health monitoring based on multiple optimization strategies, The Structural Design of Tall and Special Buildings 20 (2011), no. 7, 891-900. 